\title{
MODULAR INTERSECTION COHOMOLOGY COMPLEXES ON FLAG VARIETIES
}

\author{
GEORDIE WILLIAMSON \\ WITH AN APPENDIX BY TOM BRADEN
}

\begin{abstract}
We present a combinatorial procedure (based on the $W$-graph of the Coxeter group) which shows that the characters of many intersection cohomology complexes on low rank complex flag varieties with coefficients in an arbitrary field are given by Kazhdan-Lusztig basis elements. Our procedure exploits the existence and uniqueness of parity sheaves. In particular we are able to show that the characters of all intersection cohomology complexes with coefficients in a field on the flag variety of type $A_{n}$ for $n<7$ are given by Kazhdan-Lusztig basis elements. By results of Soergel, this implies a part of Lusztig's conjecture for $S L(n)$ with $n \leq 7$. We also give examples where our techniques fail.

In the appendix by Tom Braden examples are given of intersection cohomology complexes on the flag varities for $S L(8)$ and $S O(8)$ which have torsion in their stalks or costalks.
\end{abstract}

\section{INTRODUCTION}

Let $\mathbb{k}$ be a field of characteristic $p \geq 0$. Let $G$ be a connected reductive algebraic group over $\mathbb{C}, B \subset G$ denote a Borel subgroup of $G$ and let $(W, S)$ be the corresponding Weyl group and its simple reflections. Consider the flag variety $G / B$ with its classical (metric) topology and let $D_{\Lambda}^{b}(G / B)$ denote the bounded derived category of sheaves of $\mathbb{k}$-vector spaces on $G / B$ constructible along $B$ orbits. In $D_{\Lambda}^{b}(G / B)$ there exist the intersection cohomology sheaves $\operatorname{IC}(w)$. The sheaf $\operatorname{IC}(w)$ is supported on the closure of the Bruhat cell $B w B / B$ and its restriction to $B w B / B$ is a constant sheaf in degree $-\ell(w)$.

Let $\mathcal{H}$ be the Hecke algebra of $(W, S)$ over $\mathbb{Z}\left[v, v^{-1}\right]$ normalised so as to satisfy

$$
H_{s} H_{w}= \begin{cases}H_{s w} & \text { if } s w>w \\ \left(v-v^{-1}\right) H_{w}+H_{s w} & \text { if } s w<w \\ 1 & \end{cases}
$$


and let $\left\{\underline{H}_{w} \mid w \in W\right\}$ be the Kazhdan-Lusztig basis of $\mathcal{H}$. It satisfies $\underline{H}_{w} \in H_{w}+\oplus_{x<w} v^{-1} \mathbb{N}\left[v^{-1}\right] H_{x}$. Given a finite dimensional graded vector space $V=\oplus V_{i}$ let $P(V)=\sum\left(\operatorname{dim} V_{i}\right) v^{i}$ be its Poincaré polynomial.

The character of a sheaf $\mathcal{F} \in D_{\Lambda}^{b}(G / B)$ is the element of $\mathcal{H}$ given by

$$
\operatorname{ch}(\mathcal{F})=\sum_{w \in W} P\left(H^{*}\left(\mathcal{F}_{w}\right)\right) v^{\ell(w)} H_{w}
$$

where $\mathcal{F}_{w}$ denotes the stalk of $\mathcal{F}$ at the point in $G / B$ corresponding to $w \in W$. If $\mathbb{k}$ is of characteristic zero, a theorem of Kazhdan and Lusztig [KL80, Spr82] says that $\operatorname{ch}(\mathbf{I C}(w))=\underline{H}_{w}$. Thus the Poincaré polynomials of the stalks of the intersection cohomology sheaves are given by Kazhdan-Lusztig polynomials. It then follows that the same is true in almost all characteristics ${ }^{1}$, however for any given characteristic almost nothing is known.

It is a difficult question to determine over which fields one has $\operatorname{ch}(\mathbf{I C}(w))=\underline{H}_{w}$ and, if not, what these characters are. It has been known since the original papers of Kazhdan and Lusztig ([KL79] and [KL80]) that in non-simply laced cases the intersection cohomology complexes may have a different character in characteristic 2. (This happens, for example, in the only non-smooth Schubert variety in the flag variety of $S p(4)$.) In 2002 Braden discovered examples of Schubert varieties in simply laced types $A_{7}$ and $D_{4}$ where the character of the intersection cohomology sheaf in characteristic 2 is different to all other characteristics (see the appendix).

In this article we define combinatorially a certain subset $\sigma(W) \subset$ $W$ of separated elements and show:

Theorem 1.1. Suppose that $x \in \sigma(W)$, then $\operatorname{ch}(\mathbf{I C}(w))=\underline{H}_{w}$ for any field $\mathbb{k}$.

The determination of the characters of $\mathbf{I C}(w)$ is closely related to the decomposition theorem (see [BBD82, 6.2.5] or [dCM05, 2.1.1]). Given a simple reflection $s \in S$ let $P_{s}$ be the corresponding standard minimal parabolic subgroup and consider the quotient map

$$
G / B \stackrel{\pi_{s}}{\rightarrow} G / P_{s} \text {. }
$$

\footnotetext{
${ }^{1}$ Intersection cohomology complexes $\mathbf{I C}(X, \mathbb{Q})$ admit integral forms $\operatorname{IC}(X, \mathbb{Z})$ such that the cohomology groups of the stalks and costalks are finitely generated (see [Jut09]). If the cohomology groups of the stalks and costalks are free of $p$-torsion (which will be the case for all but finitely many primes $p$ ), one has $\mathbf{I C}(X, \mathbb{Z}) \otimes_{\mathbb{Z}}^{L} \mathbb{k} \cong \mathbf{I C}(X, \mathbb{k})$ and $\operatorname{ch}(\mathbf{I C}(X, \mathbb{Q}))=\operatorname{ch}(\mathbf{I C}(X, \mathbb{k}))$. For more details see the end of Section 3.
} 
If $\mathbb{k}$ is of characteristic zero, the decomposition theorem implies that $\pi_{s *} \mathbf{I C}(w)$ is a direct sum of shifts of intersection cohomology sheaves. This need not be true if $\mathbb{k}$ is of positive characteristic. Given $w \in W$ and $s \in S$ let $\left\{w_{1}, \ldots, w_{n}\right\}$ be the parameters of Kazhdan-Lusztig basis elements that occur with non-zero coefficient when the product $\underline{H}_{w} \underline{H}_{s}$ is expressed in the Kazhdan-Lusztig basis. Then we have:

Theorem 1.2. Suppose that $w$ and $w_{1}, \ldots, w_{n}$ lie in $\sigma(W)$. Then the decomposition theorem holds for $\pi_{s *} \mathbf{I C}(w)$; that is, $\pi_{s *} \mathbf{I C}(w)$ is isomorphic to a direct sum of shifts of intersection cohomology complexes.

Whilst being of considerable intrinsic interest, these questions are also important in representation theory. Assume that $\mathbb{k}$ is algebraically closed and that the characteristic of $\mathbb{k}$ is strictly greater than the Coxeter number of $W$. Now let $G^{\vee}$ be a semi-simple and simply connected algebraic group over $\mathbb{k}$ with maximal torus $T^{\vee} \subset G^{\vee}$ and root system dual to that of $G$ (for a choice of maximal torus $T \subset B$ ). Choose a Borel subgroup $B^{\vee} \supset T^{\vee}$ and define positive roots $R^{+} \subset$ $X\left(T^{\vee}\right)$ so that the roots corresponding to $B^{\vee}$ are those lying in $-R^{+}$. To each weight $\lambda \in X\left(T^{\vee}\right)$ one may associate a module $H^{0}(\lambda)$ which is non-zero if and only if $\lambda$ is dominant, in which case it contains a unique simple submodule $L(\lambda)$.

A conjecture of Lusztig [Lus80] expresses the characters of the simple $G^{\vee}$-modules $L(\lambda)$ in terms of the (known) characters of the modules $H^{0}(\lambda)$. A particular case of the conjecture is the following (see [Soe00]): let $\rho \in X\left(T^{\vee}\right)$ denote the half-sum of the positive roots, and let $s t=(p-1) \rho$ the Steinberg weight, then it is conjectured that,

$$
\left[H^{0}(s t+x \rho): L(s t+y \rho)\right]=h_{x, y}(1) \quad \text { for all } x, y \in W,
$$

where $h_{x, y} \in v^{-1} \mathbb{N}\left[v^{-1}\right]$ is the Kazhdan-Lusztig polynomial indexed by $x, y \in W$. A theorem of Soergel [Soe00] says that (1) is equivalent to the semi-simplicity of $\pi_{s *} \mathbf{I C}(x)$ for all $x \in W$ and $s \in S$.

Of course, in order to apply Theorems 1.1 and 1.2 it is necessary to know the set $\sigma(W)$. The essential ingredient in the calculation of $\sigma(W)$ is the $W$-graph of the Coxeter system $(W, S)$. Unfortunately, even in simple situations the $W$-graph can be very complicated and no general description is known. However, using Fokko du Cloux's program Coxeter $[\mathrm{dC}]$ it is possible to use a computer to determine the set $\sigma(W)$ for low rank Weyl groups. The simplest situation is when $\sigma(W)=W$. This only occurs in type A in low rank:

Theorem 1.3. Let $G$ be of type $A_{n}$ for $n \leq 6$. Then $\sigma(W)=W$. Hence, in all characteristics the intersection cohomology complexes have characters 
given by Kazhdan-Lusztig basis elements and the decomposition theorem holds for $\pi_{s *} \mathbf{I C}(x)$ for all $s \in S$ and $x \in W$.

It also follows that (1) holds for $G^{\vee}=S L_{n}(k)$ if $n \leq 7$ and $\mathbb{k}$ has characteristic $>n+1$.

In other types and type $A_{n}$ for $n \geq 7$ our techniques are not as effective. In most examples that we have computed $\sigma(W)$ is not the entire Weyl group. However, we are able to show that the characters are given by Kazhdan-Lusztig basis elements and verify an analogue of the decomposition theorem for many $w \in W$ (that is those $w \in$ $\sigma(W)$ ) in ranks $\leq 6$. It also seems that the elements $x \notin \sigma(W)$ (for which our methods fail) will provide an interesting source of future research.

Indeed in the appendix Braden shows that, both in type $D_{4}$ and $A_{7}$, the intersection cohomology sheaf over the integers corresponding to a minimal element in $W \backslash \sigma(W)$ has 2-torsion in the cohomology groups of its stalks or costalks (and hence $\operatorname{ch}(\mathbf{I C}(w)) \neq \underline{H}_{w}$ if the coefficients are taken to be of characteristic 2). These two examples, together with the case of dihedral groups, leads one to suspect a close relationship between $W \backslash \sigma(W)$, and those intersection cohomology complexes which have torsion in the cohomology groups of their stalks or costalks over $\mathbb{Z}$. It would be interesting to have more examples in this direction.

Let us briefly mention that, in [BM01] Braden and MacPherson give an algorithm for the calculation of the stalks of the intersection cohomology complexes with coefficients in $\mathbb{Q}$, using only data that can be obtained from the fixed points and one-dimensional orbits of a maximal torus acting on the flag variety. (This data is encoded in the so-called "moment graph" of the flag variety.) The recent paper [FW] of Fiebig and the author extends this result, showing that the moment graph of the flag variety can be used to calculate the characters of parity sheaves (a certain class of sheaves characterised by the vanishing of stalks and costalks in degrees of a fixed parity). It follows that this algorithm can be used to determine those intersection cohomology complexes which have torsion in the cohomology groups of their stalks or costalks over the integers (see Corollary 3.13). Thus the results of this paper could (at least in principle) be deduced from the moment graph. In fact, the computations of torsion in the appendix translate easily into the moment graph language and give a proof that the moment graph sheaves obtained via direct image from certain Bott-Samelson resolutions do not split as much as expected unless 2 is invertible in the coefficient ring. 
The structure of the paper is as follows. In Section 2 we review the Hecke algebra and Kazhdan-Lusztig basis in more detail and recall the $W$-graph associated to $(W, S)$. In Section 3 we discuss parity sheaves, which are our main theoretical tool. In Section 4 we define the subset $\sigma(W) \subset W$ and prove Theorems 1.1 and 1.2. In Section 5 we discuss the calculation of the sets $\sigma(W)$ via computer and give some examples of the sets $\sigma(W)$ for low rank Weyl groups.

1.1. Acknowledgments. I would like to thank Tom Braden for useful correspondence, pointing out errors in previous versions, and contributing the appendix. Both G.W. and T.B. would like to extend their gratitude to Olaf Schnürer for very detailed feedback on a previous version of this manuscript, which lead to the rewriting of Section 4, as well as smaller improvements on almost every page! We would also like to thank Simon Riche and Patrick Polo for pointing out some typos.

\section{THE HeCKE ALGEBRA AND $W$-GRAPHS}

In this section we recall the Hecke algebra and Kazhdan-Lusztig basis in slightly more detail. Up to some small changes of notation we follow [Lus03]. Let $(W, S)$ be a Coxeter system with Bruhat order $\leq$ and length function $\ell: W \rightarrow \mathbb{N}$. Given $w \in W$ we define the left and right descent set to be

$$
\mathcal{L}(w)=\{s \in S \mid s w<w\} \text { and } \mathcal{R}(w)=\{s \in S \mid w s<w\} .
$$

Recall that the Hecke algebra is the free $\mathbb{Z}\left[v, v^{-1}\right]$-module with multiplication given by

$$
H_{s} H_{w}= \begin{cases}H_{s w} & \text { if } s \notin \mathcal{L}(w), \\ \left(v-v^{-1}\right) H_{w}+H_{s w} & \text { if } s \in \mathcal{L}(w) .\end{cases}
$$

The elements $H_{w}$ are invertible and there is an involution $h \mapsto \bar{h}$ on $\mathcal{H}$ which sends $H_{w}$ to $H_{w^{-1}}^{-1}$ and $v$ to $v^{-1}$. We will call elements fixed by this involution self-dual.

There exists a basis $\left\{\underline{H}_{w}\right\}$ of $\mathcal{H}$ called the Kazhdan-Lusztig basis which is uniquely determined by requiring:

(1) the $\underline{H}_{w}$ are self-dual;

(2) $\underline{H}_{w}=\sum_{x \leq w} h_{x, w} H_{x}$ where $h_{w, w}=1$ and $h_{x, w} \in v^{-1} \mathbb{Z}\left[v^{-1}\right]$ for $x \neq w$.

The polynomials $h_{x, w}$ are (up to a renormalisation) the Kazhdan-Lusztig polynomials. One may check, for example, that $\underline{H}_{s}=H_{s}+v^{-1} H_{i d}$. 
The action of $\underline{H}_{s}$ for $s \in S$ on the Kazhdan-Lusztig basis has a particularly simple form. We denote by $\mu(x, w)$ the coefficient of $v^{-1}$ in $h_{x, w}$. Then (see [Lus03, Theorem 6.6 and Corollary 6.7]):

$$
\underline{H}_{s} \underline{H}_{w}= \begin{cases}\left(v+v^{-1}\right) \underline{H}_{w} & \text { if } s \in \mathcal{L}(w), \\ \underline{H}_{s w}+\sum_{x<w ; s \in \mathcal{L}(x)} \mu(x, w) \underline{H}_{x} & \text { if } s \notin \mathcal{L}(w) .\end{cases}
$$

Simlarly, on the right we have:

$$
\text { (3) } \underline{H}_{w} \underline{H}_{s}= \begin{cases}\left(v+v^{-1}\right) \underline{H}_{w} & \text { if } s \in \mathcal{R}(w), \\ \underline{H}_{w s}+\sum_{x<w ; s \in \mathcal{R}(x)} \mu(x, w) \underline{H}_{x} & \text { if } s \notin \mathcal{R}(w) .\end{cases}
$$

It is known if $W$ is a Weyl group (the case of interest below) then $\mu(x, w) \in \mathbb{N}$ for all $x, w \in W$.

Thus all the information about the action of $\underline{H}_{S}$ on the left and right on the Kazhdan-Lusztig basis may be encoded in a labelled graph, known as the $W$-graph. The vertices correspond to the elements of $W$ and are labelled with the left and right descent sets. There is a directed edge between $x$ and $y \in W$ if $\mu(x, y) \neq 0$, in which case the edge is labelled with the value of $\mu(x, y)$. (Strictly speaking, the graph that we define is a variant of what Kazhdan and Lusztig call a $W \times W^{\mathrm{o}}$-graph.) For more details on the Kazhdan-Lusztig basis and $W$-graphs the reader is referred to [KL79], [Hum90], [Lus03] or [Soe97].

\section{PARITY SHEAVES}

In this section we recall some basic properties of "parity sheaves" introduced in [JMW09] and motivated by [Soe00]. These are our main technical tool.

We recall briefly the setting of [JMW09]. Throughout, $\mathbb{k}$ denotes a field or complete local principal ideal domain. ${ }^{2}$ All spaces will be complex algebraic $H$-varieties, for $H$ a complex linear algebraic group. Given an $H$-space $X$, we write $D_{c}^{b}(X, \mathbb{k})$ or $D_{c}^{b}(X)$ for the bounded derived category of constructible $\mathbb{k}$-sheaves on $X$ and $D_{H}^{b}(X, \mathbb{k})$ or $D_{H}^{b}(X)$ for the bounded $H$-equivariant derived category of constructible sheaves of $\mathbb{k}$-modules on $X$ (see [BL94]). Given $\mathcal{F}$ in $D_{c}^{b}(X)$ or $D_{H}^{b}(X)$ we denote by $\mathcal{H}^{j}(\mathcal{F})$ the $j^{\text {th }}$ cohomology sheaf of $\mathcal{F}$ (which is a sheaf or equivariant sheaf of $\mathbb{k}$-modules). By abuse of language, we call objects in $D_{H}^{b}(X)$ sheaves. We denote by For : $D_{H}^{b}(X) \rightarrow$

\footnotetext{
${ }^{2}$ The case where $\mathbb{k}$ is a complete local PID will only be necessary at the end of this section to prove Theorem 3.10 and discuss the relationship between the characters of parity sheaves and the existence of torsion in the cohomology groups of the stalks or costalks of intersection cohomology complexes. If one is willing to accept these results one may assume that $\mathbb{k}$ is field throughout.
} 
$D_{c}^{b}(X)$ the forgetful functor (see [BL94]). If $H$ has finitely many orbits on $X$ then the image of the forgetful functor is contained in $D_{\Lambda}^{b}(X)$, the full subcategory of $D_{c}^{b}(X)$ consisting of sheaves whose cohomology sheaves are locally constant along $H$-orbits. The category $D_{H}^{b}(X)$ is Krull-Remak-Schmidt: an object is indecomposable if and only if its endomorphism ring is local; any object admits a decomposition into indecomposable objects; and the multiplicity of an indecomposable object as a summand of any object is independent of the chosen decomposition.

All maps will be equivariant morphisms of complex algebraic varieties. Given a map $f: X \rightarrow Y$ we have functors $f_{*}, f_{!}$from $D_{H}^{b}(X)$ to $D_{H}^{b}(Y)$ and $f^{*}, f^{!}$from $D_{H}^{b}(Y)$ to $D_{H}^{b}(X)$. Similar functors exist between $D_{c}^{b}(X)$ and $D_{c}^{b}(Y)$. On the categories $D_{H}^{b}(X), D_{H}^{b}(Y), D_{c}^{b}(X)$ and $D_{c}^{b}(Y)$ we have the Verdier duality functor, which we denote by $\mathbb{D}$. We have isomorphisms of functors $\mathbb{D} f_{*} \cong f_{!} \mathbb{D}$ and $\mathbb{D} f^{*} \cong f^{!} \mathbb{D}$. All functors $f^{*}, f^{!}, f_{!}, f_{*}, \mathbb{D}$ commute with the forgetful functor.

Now let $G$ denote a connected reductive complex algebraic group and $B \supset T$ a Borel subgroup and maximal torus. Let $W$ denote the Weyl group and $S \subset W$ the set of simple reflections corresponding to $B$. Throughout $X=G / P$, where $P$ is either $B$ or a minimal standard parabolic subgroup $P_{s}$ corresponding to $s \in S$ (i.e. $P_{s}:=\overline{B s B}$ ). We regard $X$ as a $B$-variety. Each $B$-orbit is isomorphic to an affine space and the strata are classified by $W$ if $P=B$ and $W /\langle s\rangle$ if $P=P_{s}$. Given $w \in W$ (resp. $\bar{w} \in W /\langle s\rangle$ ) we denote by $X_{w}$ (resp. $X_{\bar{w}}$ ) the stratum $B w B / B$ (resp. $B w P_{s} / P_{s}$ ), by $i_{w}: X_{w} \hookrightarrow G / B$ (resp. $i_{\bar{w}}$ : $\left.X_{\bar{w}} \hookrightarrow G / P_{s}\right)$ its inclusion and by $\underline{\mathbb{k}}_{w}$ (resp. $\underline{\mathbb{k}}_{\bar{w}}$ ) the $B$-equivariant constant sheaf on $X_{w}$ (resp. $X_{\bar{w}}$ ) with fibre $\mathbb{k}$.

For brevity, ? $\in\{*, !\}$ and $X=G / B$. A sheaf $\mathcal{F} \in D_{B}^{b}(X)$ is ?-even if $i_{w}^{?} \mathcal{F}$ is isomorphic to a direct sum of even shifts of constant sheaves $\underline{\mathbb{k}}_{w}$, for all strata $X_{w} \subset X$. A sheaf is even if it is both $*$ - and !-even. A sheaf $\mathcal{F}$ is (?-) odd if $\mathcal{F}[1]$ is (?-) even. A sheaf $\mathcal{F} \in D_{B}^{b}(X)$ is (?-)parity if we have an isomorphism $\mathcal{F} \cong \mathcal{F}_{0} \oplus \mathcal{F}_{1}$ with $\mathcal{F}_{0}$ (?-)even and $\mathcal{F}_{1}$ (?-)odd. Note that direct sums and summands of (?-)parity sheaves are (?-)parity. Entirely analagous definitions apply when $X=G / P_{s}$.

The following theorem shows that one may classify indecomposable parity sheaves on the flag variety in a similar way to intersection cohomology sheaves:

Theorem 3.1 ([JMW09, 2.9]). For any $w \in W$ (resp. $w \in W /\langle s\rangle$ ) there exists (up to isomorphism) a unique indecomposable parity sheaf $\mathcal{E}(w, k) \in$ $D_{B}^{b}(G / B)$ (resp. $\mathcal{E}(\bar{w}, k) \in D_{B}^{b}\left(G / P_{s}\right)$ ) with support contained in $\overline{X_{w}}$ (resp. $\left.\overline{X_{\bar{w}}}\right)$ and $i_{w}^{*} \mathcal{E}(w) \cong \underline{\mathbb{k}}_{w}\left[\operatorname{dim} X_{w}\right]\left(\right.$ resp. $\left.i_{\bar{w}}^{*} \mathcal{E}(\bar{w})=\underline{\mathbb{k}}_{\bar{w}}\left[\operatorname{dim} X_{\bar{w}}\right]\right)$. 
Each $\mathcal{E}(w, \mathbb{k})($ resp. $\mathcal{E}(\bar{w}, \mathbb{k}))$ is self-dual and any indecomposable parity sheaf on $G / B$ (resp. $\left.G / P_{s}\right)$ is isomorphic to $\mathcal{E}(w, \mathbb{k})[m]$ for some $w \in W$ and $m \in \mathbb{Z}($ resp. $\mathcal{E}(\bar{w}, \mathbb{k})[m]$ for some $w \in W /\langle s\rangle$ and $m \in \mathbb{Z})$.

If the context is clear we will write $\mathcal{E}(w)$ instead of $\mathcal{E}(w, \mathbb{k})$ and $\mathcal{E}(\bar{w})$ instead of $\mathcal{E}(\bar{w}, \mathbb{k})$.

Given a $*$-parity sheaf $\mathcal{E} \in D_{B}^{b}(G / B)$ and $w \in W$ we may write $i_{w}^{*} \mathcal{E} \cong V(w) \otimes_{\mathbb{k}} \underline{\mathbb{k}}_{w}$ for some finitely-generated graded free $\mathbb{k}$-module $V(w)=\oplus V(w)_{i}$. We define the character of $\mathcal{E}$ in the Hecke algebra to be

$$
\operatorname{ch}(\mathcal{E})=\sum_{i \in \mathbb{Z}, w \in W}\left(\operatorname{rk} V(w)_{i}\right) v^{\ell(w)+i} H_{w}
$$

where $\operatorname{rk} V(w)_{i}$ denotes the rank of the free $\mathbb{k}$-module $V(w)_{i}$.

Remark 3.2. If $\mathbb{k}$ is a field and $\mathcal{E}$ is a $*$-parity sheaf then it is easily seen that $\operatorname{ch}(\mathcal{E})$ agrees with the character of $\operatorname{For}(\mathcal{E}) \in D_{\Lambda}^{b}(G / B)$ as defined in the introduction.

A similar character map exists for $D_{B}^{b}\left(G / P_{s}\right)$ for a simple reflection $s \in S$. Let $\mathcal{H}_{s}$ denote the left ideal $\mathcal{H}_{\underline{H}}$ in $\mathcal{H}$. Then $\mathcal{H}_{s}$ is free with basis $H_{w} \underline{H}_{s}$ for $w \in W^{s}$, where $W^{s} \subset W$ denotes the subset of elements $w \in W$ such that $s \notin \mathcal{R}(w)$. Given a $*$-parity sheaf $\mathcal{E} \in D_{B}^{b}\left(G / P_{s}\right)$ and $w \in W^{s}$ we can write $i_{w}^{*} \mathcal{E} \cong V(w) \otimes \underline{\mathbb{K}}_{w}$ for some finitely-generated graded free $\mathbb{k}$-module $V(w)=\oplus V(w)_{i}$. We define the character of $\mathcal{E}$ to be:

$$
\operatorname{ch}(\mathcal{E})=\sum_{i \in \mathbb{Z}, w \in W^{s}}\left(\operatorname{rk} V(w)_{i}\right) v^{\ell(w)+i} H_{w} \underline{H}_{s} .
$$

For any $s \in S$ we have obvious maps given by inclusion and multiplication:

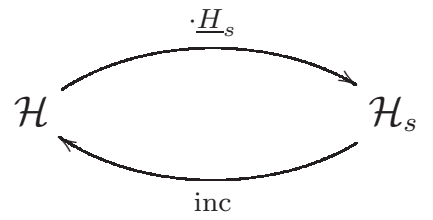

The quotient map $\pi_{s}: G / B \rightarrow G / P_{s}$ induces functors:

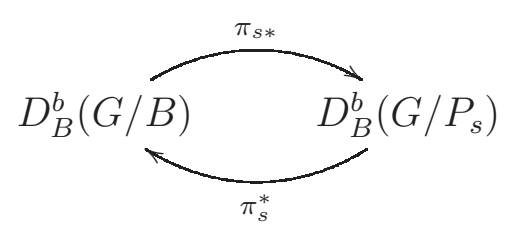

The following lemma is well-known (see [Spr82], Lemme 2.6): 
Lemma 3.3. (1) If $\mathcal{E} \in D_{B}^{b}(G / B)$ is parity, then so is $\pi_{s *} \mathcal{E}$ and

$$
\operatorname{ch}\left(\pi_{s *} \mathcal{E}\right)=\operatorname{ch}(\mathcal{E}) \underline{H}_{s} .
$$

(2) If $\mathcal{E} \in D_{B}^{b}\left(G / P_{s}\right)$ is parity, then so is $\pi_{s}^{*} \mathcal{E}$ and

$$
\operatorname{ch}\left(\pi_{s}^{*} \mathcal{E}[1]\right)=\operatorname{inc}(\operatorname{ch}(\mathcal{E})) .
$$

(3) If $\mathcal{E} \in D_{B}^{b}(G / B)$ or $D_{B}^{b}\left(G / P_{s}\right)$ is parity then

$$
\operatorname{ch}(\mathcal{E}[1])=v^{-1} \operatorname{ch}(\mathcal{E})
$$

Proof. We first show the first three relations for $*$-parity sheaves. Statement (3) is a straightforward consequence of the definitions and (2) follows from the definitions and the fact that $\pi_{s}^{-1}\left(X_{\bar{w}}\right)=X_{w} \sqcup X_{w s}$ for $w \in W$. It remains to show (1).

Let $\mathcal{E}$ be a $*$-parity sheaf. We prove (1) by induction on the number of $w \in W$ for which $i_{w}^{*} \mathcal{E} \neq 0$. If this number is one, then (by definition of $*$-parity) $\mathcal{E}$ is necessarily isomorphic to a direct sum of shifts of $i_{w !} \underline{\mathbb{K}}_{w}$, for some $w \in W$. We may assume that $\mathcal{E} \cong i_{w !} \underline{\mathbb{k}}_{w}[\ell(w)]$. Let us write $\bar{w}$ for the image of $w$ in $W /\langle s\rangle$. If $w s>w$ then $\pi_{s}$ restricts to an isomorphism $X_{w} \rightarrow X_{\bar{w}}$. Hence

$$
\pi_{s *} \mathcal{E} \cong i_{\bar{w} !} \underline{\mathbb{K}}_{\bar{w}}[\ell(w)] .
$$

If $w s<w$ then the restriction of $\pi_{s}$ to $X_{w}$ induces a (trivial) $\mathbb{C}$-bundle over $X_{\bar{w}}$, hence

$$
\pi_{s *} \mathcal{E} \cong i_{\bar{w} !} \underline{\mathbb{k}}_{\bar{w}}[\ell(w)-2]
$$

A simple calculation in the Hecke algebra then shows that in both cases

$$
\operatorname{ch}\left(\pi_{s *} \mathcal{E}\right)=\operatorname{ch}(\mathcal{E}) \underline{H}_{s}
$$

as claimed.

We now turn to the general case. We may assume without loss of generality that $\mathcal{E}$ is $*$-parity. Choose $w \in W$ so that $X_{w}$ is open in the support of $\mathcal{E}$ and let $i: \overline{\operatorname{supp} \mathcal{E}} \backslash X_{w} \hookrightarrow G / B$ denote the inclusion. Then $i_{w}^{!} \mathcal{E} \cong i_{w}^{*} \mathcal{E}$ and we have a distinguished triangle of $*$-parity sheaves

$$
i_{w !} i_{w}^{*} \mathcal{E} \rightarrow \mathcal{E} \rightarrow i_{*} i^{*} \mathcal{E} \stackrel{[1]}{\rightarrow}
$$

By induction $\pi_{s *}$ applied to the first or third term is $*$-parity and (1) holds. It follows that the same is true of $\mathcal{E}$ because $\operatorname{ch}(\mathcal{E})=$ $\operatorname{ch}\left(i_{w !} i_{w}^{*} \mathcal{E}\right)+\operatorname{ch}\left(i_{*} i^{*} \mathcal{E}\right)$ and $\operatorname{ch}\left(\pi_{s *} \mathcal{E}\right)=\operatorname{ch}\left(\pi_{s *} i_{w_{1}} i_{w}^{*} \mathcal{E}\right)+\operatorname{ch}\left(\pi_{s *} i_{*} i^{*} \mathcal{E}\right)$.

It remains to see that $\pi_{s *}$ and $\pi_{s}^{*}$ preserve the classes of parity sheaves. However this follows immediately because $\mathbb{D}$ interchanges *-parity and !-parity sheaves, $\pi_{s *} \mathbb{D} \cong \mathbb{D} \pi_{s *}$ (as $\pi_{s}$ is proper) and $\left(\pi_{s}^{*}[1]\right) \mathbb{D} \cong \mathbb{D}\left(\pi_{s}^{*}[1]\right)$ (because $\pi_{s}$ is a smooth fibration with fibres of complex dimension 1). 
Consider $G$ as a $B \times B$-space via $\left(b_{1}, b_{2}\right) \cdot g:=b_{1} g b_{2}^{-1}$. As the second copy of $B$-acts freely on $G$, the quotient equivalence ([BL94, 2.6.2]) yields an equivalence of triangulated categories:

$$
Q^{*}: D_{B}^{b}(G / B) \stackrel{\sim}{\rightarrow} D_{B \times B}^{b}(G) .
$$

Consider the inversion map $i: G \rightarrow G$. Then this is $B \times B$-equivariant with respect to the swap map $B \times B \rightarrow B \times B:\left(b_{1}, b_{2}\right) \mapsto\left(b_{2}, b_{1}\right)$. This induces an equivalence

$$
i^{*}: D_{B \times B}^{b}(G) \rightarrow D_{B \times B}^{b}(G)
$$

Consider the functor $\iota:=\left(Q^{*}\right)^{-1} i^{*} Q^{*}: D_{B}^{b}(G / B) \rightarrow D_{B}^{b}(G / B)$. Then $\iota$ commutes with $\mathbb{D}$ (see [BL94, 7.5.2]). It is easy to see that $\iota$ preserves parity sheaves and that, for a parity sheaf $\mathcal{E} \in D_{B}^{b}(G / B)$,

$$
\operatorname{ch}(\iota(\mathcal{E}))=j(\operatorname{ch}(\mathcal{E}))
$$

where $j: \mathcal{H} \rightarrow \mathcal{H}$ is the anti-involution defined by $j\left(H_{w}\right)=H_{w^{-1}}$ and $j(v)=v$.

Define endofunctors on $D_{B}^{b}(G / B)$ by

$$
(-) \vartheta_{s}:=\pi_{s}^{*} \pi_{s *}(-)[1] \text { and } \vartheta_{s}(-):=\iota \pi_{s}^{*} \pi_{s *} \iota(-)[1] \text {. }
$$

Then the functors $(-) \vartheta_{s}$ and $\vartheta_{s}(-)$ preserve parity sheaves; the shift is chosen so that $\mathbb{D}\left(\vartheta_{s} \mathcal{F}\right) \cong \vartheta_{s}(\mathbb{D} \mathcal{F})$ and $\mathbb{D}\left(\mathcal{F} \vartheta_{s}\right) \cong(\mathbb{D} \mathcal{F}) \vartheta_{s}$. By (4) and the above lemma,

$$
\operatorname{ch}\left(\mathcal{E} \vartheta_{s}\right)=\operatorname{ch}(\mathcal{E}) \underline{H}_{s} \text { and } \operatorname{ch}\left(\vartheta_{s} \mathcal{E}\right)=\underline{H}_{s} \operatorname{ch}(\mathcal{E})
$$

for parity sheaves $\mathcal{E} \in D_{B}^{b}(G / B)$.

The first result about the characters of parity sheaves is the following:

Proposition 3.4. For all $w \in W, \operatorname{ch}(\mathcal{E}(w)) \in \mathcal{H}$ is self-dual.

Proof. We proceed via induction on $\ell(w)$ with the base case being trivial. Let us fix $w$ and choose $s \in S$ with $w s<w$. By Theorem 3.1 we may write

$$
(\mathcal{E}(w s)) \vartheta_{s} \cong \mathcal{E}(w) \oplus \mathcal{G}
$$

where

$$
\mathcal{G} \cong \bigoplus_{\substack{x<w \\ \eta \in \mathbb{Z}}} \mathcal{E}(x)[\eta]^{\oplus m_{x, \eta}}
$$

The Verdier self-duality of $(\mathcal{E}(w s)) \vartheta_{s}, \mathcal{E}(w)$ and each $\mathcal{E}(x)$ for $x<w$ together with Krull-Remak-Schmidt implies

$$
m_{x,-\eta}=m_{x, \eta} .
$$


By induction, the $\operatorname{ch}(\mathcal{E}(x))$ for $x<w$ are self-dual. Hence both $\operatorname{ch}(\mathcal{G})$ and $\operatorname{ch}\left(\vartheta_{s} \mathcal{E}(s w)\right)=\underline{H}_{s} \operatorname{ch}(\mathcal{E}(s w))$ are self-dual. Thus so is $\operatorname{ch}(\mathcal{E}(w))$.

Let $s \in S$ be a simple reflection. The next proposition relates parity sheaves on $G / P_{s}$ to those on $G / B$ :

Proposition 3.5. Let $w \in W$ be such that $w s<w$ and denote by $\bar{w}$ the image of $w$ in $W /\langle s\rangle$. We have isomorphisms

$$
\pi_{s}^{*} \mathcal{E}(\bar{w})[1] \cong \mathcal{E}(w)
$$

and

$$
\pi_{s *} \mathcal{E}(w) \cong \mathcal{E}(\bar{w})[-1] \oplus \mathcal{E}(\bar{w})[1] .
$$

Proof. As $\mathcal{E}(w)$ is a direct summand of $\pi_{s}^{*} \mathcal{E}(\bar{w})[1]$ and the restriction of $\pi_{s}^{*} \mathcal{E}(\bar{w})[1]$ to $U=X_{w} \sqcup X_{w s}$ is isomorphic to a shifted constant sheaf $\underline{\mathbb{k}}_{U}[\ell(w)]$ (and hence is indecomposable) we have

$$
\operatorname{ch}(\mathcal{E}(w))=H_{w}+v^{-1} H_{w s}+\sum_{\substack{x<w \\ x \neq w s}} m_{x} H_{x}
$$

for some $m_{x} \in \mathbb{N}\left[v, v^{-1}\right]$. It follows (by considering $i_{w}^{*} \pi_{s *} \mathcal{E}(w)$ ) that

$$
\pi_{s *} \mathcal{E}(w) \cong \mathcal{E}(\bar{w})[1] \oplus \mathcal{E}(\bar{w})[-1] \oplus \mathcal{G}
$$

for some parity sheaf $\mathcal{G}$. We may also decompose

$$
\pi_{s}^{*} \mathcal{E}(\bar{w})[1] \cong \mathcal{E}(w) \oplus \mathcal{G}^{\prime} .
$$

Hence

$$
\pi_{s *} \pi_{s}^{*} \mathcal{E}(\bar{w})[1] \cong \mathcal{E}(\bar{w})[1] \oplus \mathcal{E}(\bar{w})[-1] \oplus \mathcal{G} \oplus \pi_{s *} \mathcal{G}^{\prime} .
$$

However, because $h \underline{H}_{s}=\left(v+v^{-1}\right) h$ for any $h \in \mathcal{H}_{s}$, Lemma 3.3 yields

$$
\operatorname{ch}\left(\pi_{s *} \pi_{s}^{*} \mathcal{E}(\bar{w})[1]\right)=\left(v+v^{-1}\right) \operatorname{ch}(\mathcal{E}(\bar{w}))
$$

and so $\operatorname{ch}(\mathcal{G})=\operatorname{ch}\left(\pi_{s *} \mathcal{G}^{\prime}\right)=0$. Hence $\mathcal{G}$ and $\mathcal{G}^{\prime}$ are zero.

In $D_{\Lambda}^{b}(G / B)$ and $D_{\Lambda}^{b}\left(G / P_{s}\right)$ there exist the middle perversity intersection cohomology sheaves $\mathbf{I C}(w, \mathbb{k})$ and $\mathbf{I C}(\bar{w}, \mathbb{k})$ (see [BBD82, Jut09]). The intersection cohomology complex $\mathbf{I C}(w, \mathbb{k})$ is determined up to (canonical) isomorphism by the following conditions:

IC1) $i_{x}^{*} \mathbf{I C}(w, \mathbb{k})=0$ for $x \nless w$;

IC2) $i_{w}^{*} \mathbf{I C}(w, \mathbb{k}) \cong \underline{\mathbb{k}}_{w}\left[\operatorname{dim} X_{w}\right]$;

IC3) $\mathcal{H}^{j}\left(i_{x}^{*} \mathbf{I C}(w, \mathbb{k})\right)=0$ for $x<w$ and $j \geq-\operatorname{dim} X_{x}$;

IC4) $\mathcal{H}^{j}\left(i_{x}^{l} \mathbf{I C}(w, \mathbb{k})\right)=0$ for $x<w$ and $j \leq-\operatorname{dim} X_{x}$. 
Entirely analogous conditions define $\operatorname{IC}(\bar{w}, \mathbb{k})$. If $\mathbb{k}$ is a field then the basic properties of the intersection cohomology complexes are discussed in [BBD82]. If $\mathbb{k}$ is not a field, then there are (at least) two choices of what one means by the intersection cohomology complex with coefficients in $\mathbb{k}$; the definition given above corresponds to the choice of perversity $p$ rather than $p_{+}$(in the notation of [Jut09]). We will never need the intersection cohomology complex corresponding to $p_{+}$. If $\mathbb{k}$ is a field then the intersection cohomology complexes $\mathbf{I C}(w, \mathbb{k})$ are (Verdier) self-dual. This is no longer true in general if $\mathbb{k}$ is a principal ideal domain.

The intersection cohomology complexes admit equivariant lifts $\mathbf{I C}_{B}(w, \mathbb{k}) \in D_{B}^{b}(G / B)$ and $\mathbf{I C}_{B}(\bar{w}, \mathbb{k}) \in D_{B}^{b}\left(G / P_{s}\right)$ which are uniquely determined up to isomorphism by requiring that their image under the forgetful functor is the corresponding non-equivariant intersection cohomology complex (see [BL94, 5.2]). Equivalently, $\mathbf{I C}_{B}(w, \mathbb{k})$ is the uniqe object satisfying the equivariant analogues of IC1), IC2), IC3) and IC4) above (where we replace $\mathbf{I C}(w, \mathbb{k})$ by $\left.\mathbf{I C}_{B}(w, \mathbb{k})\right)$ throughout). As with parity sheaves, we write $\mathbf{I C}(w), \mathbf{I C}_{B}(w), \mathbf{I C}(\bar{w})$ and $\mathbf{I C}_{B}(\bar{w})$ instead of $\mathbf{I C}(w, \mathbb{k})$ etc. if the ring of coefficients $\mathbb{k}$ is clear from the context.

The first relationship between parity sheaves and intersection cohomology complexes is the following:

Proposition 3.6. If $\mathbb{k}$ is a field of characteristic 0 then $\mathcal{E}(w) \cong \mathbf{I C}_{B}(w)$.

Proof. The intersection cohomology complexes $\mathbf{I C}_{B}(w)$ are simple objects in the heart of the perverse $t$-structure on $D_{B}^{b}(G / B)$ (see [BBD82]) and are therefore indecomposable. Hence we will be done by Theorem 3.1 if we can show that $\mathbf{I C}_{B}(w)$ is a parity sheaf.

Because $\mathbb{k}$ is a field of characteristic zero, $\mathbf{I C}_{B}(w) \vartheta_{s}$ is isomorphic to a direct sum of shifts of intersection cohomology complexes for all $w \in W$ (by the decomposition theorem (see [BBD82, 6.2.5] or [dCM05, 2.1.1]) and [BL94, 5.3]) together with the fact that $\pi_{s}$ is a smooth fibration). Moreover it is easy to see that if $w s<w$ then $\mathbf{I C}_{B}(w)$ is a direct summand of $\mathbf{I C}_{B}(w s) \vartheta_{s}$. Clearly $\mathbf{I C}_{B}(i d)$ is parity, and hence all $\mathbf{I C}_{B}(w)$ are parity by induction.

Now let $\mathbb{F} \subset \mathbb{k}$ denote a finite subfield of $\mathbb{k}$ and let $p$ denote the characteristic of both fields. Let $\mathbb{O}$ denote a complete discrete valuation ring of characteristic 0 with residue field $\mathbb{F}$ and field of fractions 
$\mathbb{K}$. One has functors of extension of scalars:

$$
\begin{aligned}
(-) \otimes_{\mathbb{O}}^{L} \mathbb{F}: D_{B}^{b}(G / B, \mathbb{O}) & \rightarrow D_{B}^{b}(G / B, \mathbb{F}) \\
(-) \otimes_{\mathbb{F}} \mathbb{k}: D_{B}^{b}(G / B, \mathbb{F}) & \rightarrow D_{B}^{b}(G / B, \mathbb{k}) \\
(-) \otimes_{\mathbb{O}} \mathbb{K}: D_{B}^{b}(G / B, \mathbb{O}) & \rightarrow D_{B}^{b}(G / B, \mathbb{K})
\end{aligned}
$$

By Lemma 2.19 and Proposition 2.22 in [JMW09] one has:

$$
\begin{gathered}
\mathcal{E}(y, \mathbb{O}) \otimes_{\mathbb{O}} \mathbb{K} \text { and } \mathcal{E}(y, \mathbb{O}) \otimes_{\mathbb{O}}^{L} \mathbb{F} \text { are parity sheaves; } \\
\qquad \mathcal{E}(y, \mathbb{O}) \otimes_{\mathbb{O}}^{L} \mathbb{F} \cong \mathcal{E}(y, \mathbb{F}) .
\end{gathered}
$$

Lemma 3.7. For all $y \in Y$ we have

$$
\operatorname{ch}(\mathcal{E}(y, \mathbb{F}))=\operatorname{ch}(\mathcal{E}(y, \mathbb{O}))=\operatorname{ch}\left(\mathcal{E}(y, \mathbb{O}) \otimes_{\mathbb{O}} \mathbb{K}\right) .
$$

Similarly, if $\mathcal{G}$ is a $*$-parity sheaf with coefficients in $\mathbb{F}$ then

$$
\operatorname{ch}(\mathcal{G})=\operatorname{ch}\left(\mathcal{G} \otimes_{\mathbb{F}} \mathbb{k}\right) .
$$

Proof. Fix $x \in W$. By definition of $*$-parity we can write $i_{x}^{*} \mathcal{E}(y, \mathbb{O})=$ $V(x) \otimes \underline{\mathbb{O}}_{x}$ for some finitely generated graded free $\mathbb{O}$-module $V(x)$. Because the stalks of $\mathbb{O}_{x}$ are free (and hence flat) and $i^{*}$ commutes with extension of scalars we have isomorphisms

$$
\begin{aligned}
i_{x}^{*}\left(\mathcal{E}(y, \mathbb{O}) \otimes_{\mathbb{O}}^{L} \mathbb{F}\right) \cong\left(V(x) \otimes_{\mathbb{O}} \mathbb{F}\right) \otimes_{\mathbb{F}} \underline{\mathbb{F}}_{x}, \\
i_{x}^{*}\left(\mathcal{E}(y, \mathbb{O}) \otimes_{\mathbb{O}}^{L} \mathbb{K}\right) \cong\left(V(x) \otimes_{\mathbb{O}} \mathbb{K}\right) \otimes_{\mathbb{K}} \underline{\mathbb{K}}_{x} .
\end{aligned}
$$

Hence $\operatorname{ch}\left(\mathcal{E}(y, \mathbb{O}) \otimes_{\mathbb{O}} \mathbb{F}\right)=\operatorname{ch}(\mathcal{E}(y, \mathbb{O}))=\operatorname{ch}\left(\mathcal{E}(y, \mathbb{O}) \otimes_{\mathbb{O}} \mathbb{K}\right)$. The first statement now follows from (7). The proof of the second statement is entirely analogous.

Lemma 3.8. For all $y \in W$ we have

$$
\mathcal{E}(y, \mathbb{F}) \otimes_{\mathbb{F}} \mathbb{k} \cong \mathcal{E}(y, \mathbb{k}) .
$$

Proof. Firstly, $\mathcal{E}(y, \mathbb{F}) \otimes_{\mathbb{F}}^{L} \mathbb{k}$ is parity by [JMW09, Lemma 2.19] and so we have to show that $\mathcal{E}(y, \mathbb{F}) \otimes_{\mathbb{F}} \mathbb{K}$ is indecomposable. Using [JMW09, Proposition 2.4] one deduces easily that if we set $A=\operatorname{End}(\mathcal{E}(y, \mathbb{F}))$ then $\operatorname{End}\left(\mathcal{E}(y, \mathbb{F}) \otimes_{\mathbb{F}}^{L} \mathbb{k}\right) \cong A \otimes_{\mathbb{F}} \mathbb{k}$. Hence we will be done if we can show that $A \otimes_{\mathbb{F}} \mathbb{k}$ is a local ring which is the case if $A / \operatorname{rad} A \cong$ $\mathbb{F}$ (where $\operatorname{rad} A$ denotes the Jacobsen radical of $A$ ). However by [JMW09, Corollary 2.6] we have a surjection

$$
\phi: A=\operatorname{End}(\mathcal{E}(y, \mathbb{F})) \rightarrow \operatorname{End}\left(i_{y}^{*} \mathcal{E}(y, \mathbb{F})\right)=\mathbb{F}
$$

and therefore $\operatorname{rad} A=\operatorname{ker} \phi$ because $A$ is a local ring. It follows that $A / \operatorname{rad} A \cong \mathbb{F}$ as claimed.

\footnotetext{
${ }^{3}$ By the stalk of an equivariant sheaf or complex on $X$ we mean the stalk of the corresponding sheaf or complex on the Borel construction of $X$, see [BL94].
} 
It follows that the characters of the indecomposable parity sheaves only depend on the characteristic:

Corollary 3.9. For all $y \in W$ we have

$$
\operatorname{ch}(\mathcal{E}(y, \mathbb{F}))=\operatorname{ch}(\mathcal{E}(y, \mathbb{k})) .
$$

A simple and useful consequence of the above results is the following:

Theorem 3.10. For any $y \in W$ we have

$$
\operatorname{ch}(\mathcal{E}(y, \mathbb{k}))=\sum_{x \in W} Q_{x y} \underline{H}_{x}
$$

for some Laurent polynomials $Q_{x y} \in \mathbb{N}\left[v, v^{-1}\right]$.

Proof. By Corollary 3.9 it is enough to prove the result for $\operatorname{ch}(\mathcal{E}(y, \mathbb{F}))$. By Lemma 3.7 we have

$$
\operatorname{ch}(\mathcal{E}(y, \mathbb{F}))=\operatorname{ch}(\mathcal{E}(y, \mathbb{O}))=\operatorname{ch}\left(\mathcal{E}(y, \mathbb{O}) \otimes_{\mathbb{O}} \mathbb{K}\right) .
$$

Now (6) together with Theorem 3.1 allows us to write

$$
\mathcal{E}(y, \mathbb{O}) \otimes_{\mathbb{O}} \mathbb{K} \cong \bigoplus_{x \in W} V(x, y) \otimes \mathcal{E}(x, \mathbb{K})
$$

for some finite dimensional graded vector spaces $V(x, y)=\bigoplus V(x, y)_{i}$. Hence we have

$$
\operatorname{ch}(\mathcal{E}(y, \mathbb{F}))=\operatorname{ch}\left(\mathcal{E}(y, \mathbb{O}) \otimes_{\mathbb{O}}^{L} \mathbb{F}\right)=\sum_{x \in W} Q_{x y} \operatorname{ch}(\mathcal{E}(x, \mathbb{K}))
$$

where $Q_{x y}=\sum v^{i} \operatorname{dim} V(x, y)_{i}$. The result then follows from Propositions 3.11 and 3.6 which imply that $\operatorname{ch}(\mathcal{E}(x, \mathbb{K}))=\underline{H}_{x}$.

In the last part of this section we establish a relation between the characters of the indecomposable parity sheaves with coefficients in $\mathbb{F}$, and the presence of torsion in the cohomology groups of the stalks or costalks of intersection cohomology complexes over $\mathbb{O}$ or $\mathbb{Z}$.

In the following, when we say that the stalk of a sheaf (= complex) is torsion free or has torsion, we mean that the corresponding statement is true for the direct sum of the cohomology groups of the stalk. ${ }^{4}$

Proposition 3.11. For any $w \in W$, the following are equivalent:

\footnotetext{
${ }^{4}$ For example, the statement that a sheaf $\mathcal{F}$ has torsion free stalks means that all cohomology groups of the stalks of $\mathcal{F}$ are torsion free, whereas the statement the stalk of $\mathcal{F}$ at $x$ has torsion means that there exists a cohomology group of the stalk of $\mathcal{F}$ at $x$ which has torsion.
} 
(1) $\mathrm{IC}(w, \mathbb{O})$ has torsion free stalks and costalks;

(2) $\mathbf{I C}_{B}(w, \mathbb{O}) \cong \mathcal{E}(w, \mathbb{O})$;

(3) $\mathbf{I C}_{B}(w, \mathbb{F}) \cong \mathcal{E}(w, \mathbb{F})$;

(4) $\operatorname{ch}(\mathcal{E}(w, \mathbb{O}))=\operatorname{ch}(\mathcal{E}(w, \mathbb{F}))=\underline{H}_{w}$.

Proof. $(1) \Rightarrow(2)$ : Because $\mathbb{K}$ is flat as an $\mathbb{O}$-module $\mathbf{I C}(w, \mathbb{O}) \otimes_{\mathbb{O}} \mathbb{K}$ satisfies the conditions IC1) - IC4) and hence we have an isomorphism

$$
\mathbf{I C}(w, \mathbb{O}) \otimes_{\mathbb{O}} \mathbb{K} \cong \mathbf{I C}(w, \mathbb{K}) .
$$

Now assume that $\mathbf{I C}(w, \mathbb{O})$ has torsion free stalks and costalks and let ? $\in\{!, *\}$. Then $\mathcal{H}^{j}\left(i_{x}^{?} \mathbf{I C}(w, \mathbb{O})\right)$ is a local system with torsion free stalks, and hence is isomorphic to a direct sum of trivial local systems, because $X_{x}$ is simply connected. Now $\mathcal{H}^{j}$ commutes with the forgetful functor, and hence $\mathcal{H}^{j}\left(i_{x}^{?} \mathbf{I C}_{B}(w, \mathbb{O})\right)$ is isomorphic to a direct sum of the $B$-equivariant constant sheaves $\mathbb{O}_{x}$ (the forgetful functor from $B$-equivariant local systems on $X_{x}$ to local systems is fully faithful because $B$ is connected). For all $x \in W$ we have isomorphisms

$\mathcal{H}^{j}\left(i_{x}^{?} \mathbf{I C}_{B}(w, \mathbb{O})\right) \otimes_{\mathbb{O}} \mathbb{K} \cong \mathcal{H}^{j}\left(i_{x}^{?}\left(\mathbf{I C}_{B}(w, \mathbb{O}) \otimes_{\mathbb{O}} \mathbb{K}\right)\right) \cong \mathcal{H}^{j}\left(i_{x}^{?} \mathbf{I C}_{B}(w, \mathbb{K})\right)$

(we use that $i_{x}^{?}(-)$ commutes with extension of scalars) and the last group vanishes if $j \neq \ell(w) \bmod 2$ by Proposition 3.6. Hence

$$
\mathcal{H}^{j}\left(i_{x}^{?} \mathbf{I C}_{B}(w, \mathbb{O})\right)=0 \text { for } j \neq \ell(w) \bmod 2 .
$$

By a standard decalage argument (see [JMW09, Section 2.2]) it follows that $i_{x}^{?} \mathbf{I C}_{B}(w, \mathbb{O})$ is isomorphic to a direct sum of even shifts of the $B$-equivariant constant sheaf $\underline{\mathbb{O}}_{x}[\ell(w)]$. In particular, $\mathbf{I C}_{B}(w, \mathbb{O})$ is a parity sheaf. Now, intersection cohomology complexes are indecomposable (for example, because $\mathbb{O}_{w}$ is indecomposable and $\left(i_{w}\right)$ !* is fully-faithful [Jut09, Proposition 2.29]). Hence $\operatorname{IC}_{B}(w, \mathbb{O}) \cong \mathcal{E}(w, \mathbb{O})$ by Theorem 3.1.

$(2) \Rightarrow(1)$ : Suppose $\mathbf{I C}_{B}(w, \mathbb{O}) \cong \mathcal{E}(w, \mathbb{O})$ and choose $? \in\{!, *\}$. By definition of $\mathcal{E}(w, \mathbb{O}), i_{x}^{?} \mathcal{E}(w, \mathbb{O})$ is isomorphic to a direct sum of shifted $B$-equivariant local systems $\underline{\mathbb{O}}_{x}$. Hence $i_{x}^{\text {F }} \operatorname{For}\left(\mathbf{I C}_{B}(w, \mathbb{O})\right) \cong$ $i_{x}^{?} \mathbf{I C}(w, \mathbb{O})$ is also isomorphic to a direct sum of shifted local systems with torsion free stalks. Hence the stalks and costalks of $\mathbf{I C}(w, \mathbb{O})$ are torsion free.

$(1)+(2) \Rightarrow(3)$ : If the stalks of the cohomology sheaves of $i_{x}^{?} \mathbf{I C}(w, \mathbb{O})$ are torsion free, then we have

$$
\mathcal{H}^{j}\left(i_{x}^{?} \mathbf{I C}(w, \mathbb{O}) \otimes_{\mathbb{O}}^{L} \mathbb{F}\right) \cong \mathcal{H}^{j}\left(i_{x}^{?} \mathbf{I C}(w, \mathbb{O})\right) \otimes_{\mathbb{O}} \mathbb{F} .
$$


Hence, $\mathbf{I C}(w, \mathbb{O}) \otimes_{\mathbb{O}}^{L} \mathbb{F} \cong \mathbf{I C}(w, \mathbb{F})$. Hence

$$
\mathbf{I C}_{B}(w, \mathbb{F}) \cong \mathbf{I C}_{B}(w, \mathbb{O}) \otimes_{\mathbb{O}}^{L} \mathbb{F} \cong \mathcal{E}(w, \mathbb{O}) \otimes_{\mathbb{O}}^{L} \mathbb{F} \cong \mathcal{E}(w, \mathbb{F})
$$

with the last isomorphism following by (7).

$(3) \Rightarrow(4)$ : Define

$$
h:=\operatorname{ch}(\mathcal{E}(w, \mathbb{F})) \in \mathcal{H} .
$$

Then $h$ is a self-dual element of the Hecke algebra (by Proposition 3.4). Let us write

$$
h=\sum_{x \in W} a_{x} H_{x}
$$

for some $a_{x} \in \mathbb{N}\left[v, v^{-1}\right]$. If $\mathcal{E}(w, \mathbb{F}) \cong \mathbf{I C}_{B}(w, \mathbb{F})$ then we can translate the defining properties of the intersection cohomology complex given above: IC1) gives $a_{x}=0$ for $x \nless w$; IC2) gives $a_{w}=1$; and IC3) gives $a_{x} \in v^{-1} \mathbb{N}\left[v^{-1}\right]$ for $x<w$. Hence $h=\underline{H}_{w}$ by the uniqueness of the Kazhdan-Lusztig basis.

$(4) \Rightarrow(2)$ : By definition, $\mathcal{E}(w, \mathbb{O})$ is self-dual, supported on the closure $\overline{X_{w}}$ and, for $? \in\{!, *\}, i_{x}^{?} \mathcal{E}(w, \mathbb{O})$ is isomorphic to $\mathbb{Q}_{w}\left[\operatorname{dim} X_{w}\right]$ if $x=w$ and is isomorphic to a direct sum of shifts of equivariant constant sheaves in general. Using that $\mathbb{O}_{x}[\ell(x)]$ is self-dual and $\mathbb{D} i_{x}^{*} \cong i_{x}^{!} \mathbb{D}$ we see that $\mathcal{E}(w, \mathbb{O})$ satisfies the above conditions to be the (equivariant) intersection cohomology complex if it satisfies IC3) which is the statement that, for all $x<w$,

$$
\mathcal{H}^{j}\left(i_{x}^{*} \mathcal{E}(w, \mathbb{O})\right)=0 \quad \text { for } j \geq-\ell(x) .
$$

This is the case if and only if, when we write $\operatorname{ch}(\mathcal{E}(w, \mathbb{O}))=\sum_{x \in W} b_{x} H_{x}$ we have $b_{x} \in v^{-1} \mathbb{N}\left[v^{-1}\right]$ for $x<w$. However, this is clearly satisfied if $\operatorname{ch}(\mathcal{E}(w, \mathbb{O}))=\underline{H}_{w}$.

The above proposition has an obvious analogue on $G / P_{s}$ for $s \in S$. (One can check that the above proof also applies in this situation.)

Proposition 3.12. Suppose that $w \in W$ with $w s<w$. Then the following are equivalent:

(1) $\operatorname{IC}(\bar{w}, \mathbb{O})$ has torsion free stalks and costalks;

(2) $\mathbf{I C}_{B}(\bar{w}, \mathbb{O}) \cong \mathcal{E}(\bar{w}, \mathbb{O})$;

(3) $\operatorname{IC}_{B}(\bar{w}, \mathbb{F}) \cong \mathcal{E}(\bar{w}, \mathbb{F})$;

(4) $\operatorname{ch}(\mathcal{E}(\bar{w}, \mathbb{O}))=\operatorname{ch}(\mathcal{E}(\bar{w}, \mathbb{F}))=\underline{H}_{w}$.

We finish this section with a corollary of the above results which justifies a number of statements made in the introduction.

Corollary 3.13. The following are equivalent:

(1) the stalks and costalks of $\mathbf{I C}(x, \mathbb{Z})$ are free of $p$-torsion; 
(2) $\mathcal{E}(w, \mathbb{k}) \cong \mathbf{I C}_{B}(w, \mathbb{k})$;

(3) $\operatorname{ch}(\mathcal{E}(w, \mathbb{k})) \cong \underline{H}_{w}$.

Proof. Consider the statements:

(2') $\mathcal{E}(w, \mathbb{F}) \cong \mathbf{I C}_{B}(w, \mathbb{F})$;

(3') $\operatorname{ch}(\mathcal{E}(w, \mathbb{F})) \cong \underline{H}_{w}$.

Clearly (3) $\Leftrightarrow\left(3^{\prime}\right)$ by Corollary 3.9. We first show $(1) \Leftrightarrow\left(2^{\prime}\right) \Leftrightarrow\left(3^{\prime}\right)$ and then $(2) \Leftrightarrow\left(2^{\prime}\right)$.

Because $\mathbb{O}$ is a flat $\mathbb{Z}$-module one has an isomorphism

$$
\mathbf{I C}(w, \mathbb{O}) \cong \mathbf{I C}(w, \mathbb{Z}) \otimes_{\mathbb{Z}} \mathbb{O} .
$$

Hence the stalks or costalks of $\mathbf{I C}(w, \mathbb{O})$ will have torsion if and only if the stalks or costalks of $\mathbf{I C}(w, \mathbb{Z})$ have $p$-torsion. Hence we have that (1), (2') and (3') are equivalent by Proposition 3.11.

We can check whether $\mathcal{E}(w, \mathbb{F})$ is isomorphic to $\mathbf{I C}_{B}(w, \mathbb{F})$ (resp. $\mathcal{E}(w, \mathbb{k})$ is isomorphic to $\left.\mathbf{I C}_{B}(w, \mathbb{k})\right)$ by verifying conditions IC1), IC20, IC3) and IC4) given at the start of this section. As $\mathbb{F} \subset \mathbb{k}$ is flat, it is easy to see that these conditions will be verified for $\mathcal{E}(w, \mathbb{F})$ if and only if they are verified for $\mathcal{E}(w, \mathbb{k})$. Hence $(2)$ and $\left(2^{\prime}\right)$ are equivalent.

\section{SEPARATED ELEMENTS}

In this section $\mathbb{k}$ denotes a field of charactheristic $p$ and we fix a set of representatives $\{\mathcal{E}(w) \mid w \in W\}$ for the isomorphism classes of indecomposable parity sheaves on $G / B$ with coefficients in $\mathbb{k}$. We would like to investigate when their characters are equal to the Kazhdan-Lusztig basis. By results of the previous section, the characters of indecomposable parity sheaves yield a self-dual basis of $\mathcal{H}$ with certain positivity properties which are shared by the KazhdanLusztig basis. For example, by the results of the previous section, if we express $\operatorname{ch}(\mathcal{E}(x)) \underline{H}_{S}$ and $\underline{H}_{S} \operatorname{ch}(\mathcal{E}(x))$ in the basis $\{\operatorname{ch}(\mathcal{E}(w)) \mid w \in$ $W\}$ then the coefficients belong to $\mathbb{N}\left[v, v^{-1}\right]$. In this section we investigate to what extent these properties already determine the basis. ${ }^{5}$

We start by introducing some notation. Given $a, a^{\prime} \in \mathbb{Z}\left[v, v^{-1}\right]$ write

$$
a \leq a^{\prime} \Leftrightarrow a^{\prime}-a \in \mathbb{N}\left[v, v^{-1}\right]
$$

\footnotetext{
${ }^{5}$ One can also prove that, if one expresses any product $\operatorname{ch}(\mathcal{E}(x)) \operatorname{ch}(\mathcal{E}(y))$ in the basis $\{\operatorname{ch}(\mathcal{E}(w)) \mid w \in W\}$ then the coefficients are Laurent polynomials with positive coefficients. We will not make use of this stronger positivity property below.
} 
Given $h, h^{\prime} \in \mathcal{H}$ we expand them in the Kazhdan-Lusztig basis as $h=\sum a_{x} \underline{H}_{x}$ and $h^{\prime}=\sum a_{x}^{\prime} \underline{H}_{x}$ and write

$$
h \leq h^{\prime} \Leftrightarrow a_{x} \leq a_{x}^{\prime} \text { for all } x \in W .
$$

Using the fact that $0 \leq \underline{H}_{w} \underline{H}_{s}$ and $0 \leq \underline{H}_{s} \underline{H}_{w}$ for all $w \in W$ and $s \in$ (which follows from (2) and (3) in Section 2) we have

$$
h \leq h^{\prime} \Rightarrow h \underline{H}_{s} \leq h^{\prime} \underline{H}_{s} \text { and } \underline{H}_{s} h \leq \underline{H}_{s} h^{\prime} .
$$

Given polynomials $a^{i}=\sum_{j} a_{j}^{i} v^{j} \in \mathbb{Z}\left[v, v^{-1}\right]$ where $i$ runs over some finite indexing set $I$ define

$$
\min _{i \in I}\left\{a^{i}\right\}=\sum_{j}\left(\min _{i \in I}\left\{a_{j}^{i}\right\}\right) v^{j} .
$$

For example $\min \left\{v^{-2}+1+2 v^{2}, 4 v^{-2}+v^{2}\right\}=v^{-2}+v^{2}$. Similarly, if $h^{i}=\sum_{x \in W} a_{x}^{i} \underline{H}_{x}$ is a family of elements of $\mathcal{H}$ indexed by $i \in I$ (again assumed finite) set

$$
\min _{i \in I}\left\{h^{i}\right\}=\sum_{x \in W} \min _{i \in I}\left\{a_{x}^{i}\right\} \underline{H}_{x} .
$$

Clearly if $a \in \mathbb{Z}\left[v, v^{-1}\right]$ satisfies $a \leq a^{i}$ for all $i \in I$ then $a \leq \min _{i \in I}\left\{a^{i}\right\}$. Similarly, if $h \in \mathcal{H}$ satisfies $h \leq h^{i}$ for all $i \in I$ then $h \leq \min _{i \in I}\left\{h^{i}\right\}$.

We now define elements $\underline{B}_{y} \in \mathcal{H}$ for each $y \in W$. We define these elements inductively as follows:

(1) $\underline{B}_{i d}=\underline{H}_{i d}=H_{i d}$

(2) Let $y \in W$ and assume that we have defined $\underline{B}_{x}$ for all $x<y$. Define

$$
\underline{B}_{y}=\min \left\{\min _{s \in \mathcal{L}(y)}\left\{\underline{H}_{s} \underline{B}_{s y}\right\}, \min _{t \in \mathcal{R}(y)}\left\{\underline{B}_{y t} \underline{H}_{t}\right\}\right\} .
$$

For example $\underline{B}_{s}=\underline{H}_{s}$ for all $s \in S$ and $\underline{B}_{s t}=\underline{H}_{s t}$ for any $s \neq t \in S$.

By induction, all the information needed to evaluate this formula is encoded in the $W$-graph (using (2) and (3) in Section 2). Another immediate consequence of this formula is that if we define polynomials $D_{x y} \in \mathbb{Z}\left[v, v^{-1}\right]$ by

$$
\underline{B}_{y}=\sum_{x} D_{x y} \underline{H}_{x}
$$

then $D_{x y} \in \mathbb{N}\left[v, v^{-1}\right], D_{x y}=0$ unless $x \leq y$ and $D_{y y}=1$. In particular, $\left\{\underline{B}_{y} \mid y \in W\right\}$ is a basis of $\mathcal{H}$.

Definition 1. Let $y \in W$, if $\underline{B}_{y}=\underline{H}_{y}$ we say that $y$ is separated. We denote the set of separated elements in $W$ by $\sigma(W)$. 
We will see in Proposition 4.3 below that for any separated $y \in W$ we have that $\operatorname{ch}(\mathcal{E}(y))=\underline{H}_{y}$ for all fields of coefficients $\mathbb{k}$. This is the content of Theorem 1.1 of the introduction.

Example 4.1. Let $W$ be a dihedral group:

$$
D_{n}=\left\langle s, t \mid s^{2}=t^{2}=(s t)^{n}=i d\right\rangle .
$$

Then one may show that $\underline{B}_{x}=\underline{H}_{x}$ if and only if $x \in\left\{i d, s, t, s t, t s, w_{0}\right\}$. In particular, $A_{2}$ and $A_{1} \times A_{1}$ are the only rank two Weyl groups in which $\sigma(W)=W$.

With the above notions in hand, we now revisit the problem of calculating the characters of indecomposable parity sheaves.

Lemma 4.2. Let $\mathcal{F}$ be a parity sheaf. Then for any direct summand $\mathcal{G}$ of $\mathcal{F}$ one has

$$
\operatorname{ch}(\mathcal{G}) \leq \operatorname{ch}(\mathcal{F})
$$

Proof. This is immediate from the fact that, by Theorems 3.1 and 3.10, the characters of parity sheaves are $\mathbb{N}\left[v, v^{-1}\right]$-linear combinations of the Kazhdan-Lusztig basis $\left\{\underline{H}_{x} \mid x \in W\right\}$.

Proposition 4.3. With coefficients $\mathbb{k}$ in any field we have

$$
\operatorname{ch}(\mathcal{E}(y)) \leq \underline{B}_{y}
$$

for all $y \in W$.

In particular, for all $y \in \sigma(W)$ we have $\operatorname{ch}(\mathcal{E}(y))=\underline{H}_{y}$.

Proof. Clearly $\operatorname{ch}(\mathcal{E}(i d))=\underline{H}_{i d}=\underline{B}_{i d}$ and so we may assume by induction that the proposition is true for all $x<y$. For all $s \in \mathcal{L}(y)$ we know that $\mathcal{E}(y)$ occurs as a direct summand of $\vartheta_{s} \mathcal{E}(s y)$. Now

$$
\operatorname{ch}(\mathcal{E}(y)) \leq \operatorname{ch}\left(\vartheta_{s} \mathcal{E}(s y)\right)=\underline{H}_{s} \operatorname{ch}(\mathcal{E}(s y)) \leq \underline{H}_{s} \underline{B}_{s y} .
$$

by Lemma 4.2, Lemma 3.3 and (9) respectively. Similarly, for all $t \in$ $\mathcal{R}(y), \mathcal{E}(y)$ occurs as a direct summand of $\mathcal{E}(y t) \vartheta_{t}$ and so

$$
\operatorname{ch}(\mathcal{E}(y)) \leq \underline{B}_{y t} \underline{H}_{t} .
$$

Intersecting all of these conditions (see the remarks at the beginning of this section) gives

$$
\operatorname{ch}(\mathcal{E}(y)) \leq \min \left\{\min _{s \in \mathcal{L}(y)}\left\{\underline{H}_{s} \underline{B}_{s y}\right\}, \min _{t \in \mathcal{R}(y)}\left\{\underline{B}_{y t} \underline{H}_{t}\right\}\right\}=\underline{B}_{y} .
$$

The last statement of the proposition is immediate: if $\underline{B}_{y}=\underline{H}_{y}$ then $0 \leq \operatorname{ch}(\mathcal{E}(y)) \leq \underline{B}_{y}$ forces $\operatorname{ch}(\mathcal{E}(y))=\underline{H}_{y}$ because $\operatorname{ch}(\mathcal{E}(y))$ is nonzero. 
Remark 4.4. One may show that if $G$ is of rank 2 with Weyl group $W$ (a dihedral group), then the Schubert varieties corresponding to elements $w \in \sigma(W)$ are smooth. Hence Proposition 4.3 does not give any new information in this case.

It remains to prove Theorem 1.2 from the introduction. In fact we prove a more precise result. Fix $w \in W$ and $s \in S$ with $w s>w$. By (3) in Section 2 we can write:

$$
\underline{H}_{w} \underline{H}_{s}=\underline{H}_{w s}+\sum_{z s<z<w} \mu(z, w) \underline{H}_{z} .
$$

Define $A(w, s)=\{w s\} \cup\{z \in W \mid z s<z<w, \mu(z, w) \neq 0\}$.

Proposition 4.5. Suppose that $w$ and each element of $A(w, s)$ belongs to $\sigma(W)$. Then one has an isomorphism

$$
\pi_{s *} \mathcal{E}(w) \cong \mathcal{E}(\bar{w}) \oplus \bigoplus_{z s<z<w} \mathcal{E}(\bar{z})^{\oplus \mu(z, w)} .
$$

Proof. By Theorem 3.1 we have a decomposition

$$
\pi_{s *} \mathcal{E}(w) \cong \bigoplus_{\substack{z \in W ; z s<z \\ \nu \in \mathbb{Z}}} \mathcal{E}(\bar{z})[\nu]^{\oplus m_{z, \nu}}
$$

for certain natural numbers $m_{z, \nu} \in \mathbb{N}$. Applying $\pi_{s}^{*}$ and using Proposition 3.5 we obtain

$$
\mathcal{E}(w) \vartheta_{s}=\pi_{s}^{*} \pi_{s *} \mathcal{E}(w)[1] \cong \bigoplus_{\substack{z \in W ; z<<z \\ \nu \in \mathbb{Z}}} \mathcal{E}(z)[\nu]^{\oplus m_{z, \nu}}
$$

By assumption, $w \in \sigma(W)$ and so $\operatorname{ch}(\mathcal{E}(w))=\underline{H}_{w}$. By Lemmas 4.2 and 3.3 we have, for any $z$,

$$
\operatorname{ch}\left(\mathcal{E}(z)[\nu]^{\oplus m_{z, \nu}}\right) \leq \operatorname{ch}\left(\mathcal{E}(w) \vartheta_{s}\right)=\underline{H}_{w} \underline{H}_{s} .
$$

In view of (10) we conclude that $m_{z, \nu}=0$ unless $\nu=0$ and that $m_{z, \nu} \neq 0$ implies $z \in A(w, s)$. On the other hand, our assumptions guarantee that $\operatorname{ch}(\mathcal{E}(z))=\underline{H}_{z}$ for all $z \in A(w, s)$. Hence $m_{w s, 0}=$ 1 and $m_{z, 0}=\mu(z, w)$ for $w s \neq z \in A(w, s)$. The proposition then follows.

If $w \in \sigma(W)$ then $\operatorname{ch}(\mathcal{E}(w))=\underline{H}_{w}$ and so $\mathcal{E}(w, \mathbb{k}) \cong \mathbf{I C}_{B}(w, \mathbb{k})$ by Corollary 3.13. Hence (under the same assumptions as in the above proposition) we have an isomorphism

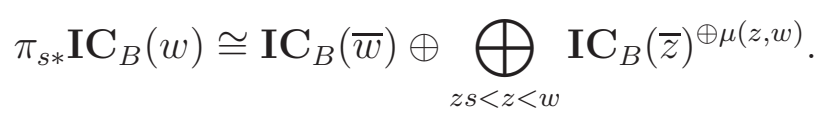


Applying the forgetful functor yields

$$
\pi_{s *} \mathbf{I C}(w) \cong \mathbf{I C}(\bar{w}) \oplus \bigoplus_{z s<z<w} \mathbf{I C}(\bar{z})^{\oplus \mu(z, w)} .
$$

On the other hand, if $w s<w$ then, by Proposition 3.5, we have

$$
\pi_{s *} \mathcal{E}(w) \cong \mathcal{E}(\bar{w})[1] \oplus \mathcal{E}(\bar{w})[-1] .
$$

Now, if $w \in \sigma(W)$, then $\mathcal{E}(w) \cong \mathbf{I C}_{B}(w)$ as above and

$$
\underline{H}_{w}=\operatorname{ch}(\mathcal{E}(w))=\operatorname{ch}\left(\pi_{s}^{*} \mathcal{E}(\bar{w})[1]\right)=\operatorname{ch}(\mathcal{E}(\bar{w}))
$$

by Propositions 4.3 and 3.5 and Lemma 3.3. Proposition 3.12 then gives $\mathcal{E}(\bar{w}) \cong \mathbf{I C}_{B}(\bar{w})$. Hence

$$
\pi_{*} \mathbf{I C}(w) \cong \mathbf{I C}(\bar{w})[1] \oplus \mathbf{I C}(\bar{w})[-1] .
$$

This proves Theorem 1.2 from the introduction.

\section{Results of COMPuter CAlculations}

In this section we give some examples of the sets $\sigma(W) \subset W$ and the basis $\left\{\underline{B}_{x}\right\}$ for low rank Weyl groups. As is clear from its definition, the only information needed to calculate the basis $\left\{\underline{B}_{x}\right\}$ is the Coxeter system $(W, S)$ and its $W$-graph described in Section 2 . However, no general description of the $W$-graph is known (for descriptions of some subgraphs see [LS81] and [Ker83] and for a description of the computational aspects of the problem see [dC02] and [OK95]).

Thus, in order to calculate the basis $\left\{\underline{B}_{x}\right\}$ (and hence $\sigma(W)$ ) we have to restrict ourselves to examples. This involves two steps:

(1) calculation of the $W$-graph of $(W, S)$, and

(2) calculation of the basis $\left\{\underline{B}_{x}\right\}$ using the $W$-graph.

Step 1) is computationally quite difficult, especially when the Weyl group is large. Luckily there exists the program Coxeter written by Fokko du Cloux [dC], which calculates the $W$-graph very efficiently. Step 2) is then relatively straightforward. A crude implementation in Magma (whose routines for handling Coxeter groups proved very useful) as well as the $W$-graphs obtained from Coxeter are available at:

http://people.maths.ox.ac.uk/williamsong/torsion/

This site also contains a complete description of the basis elements $\underline{B}_{x}$ for $x \in W$ and sets $\sigma(W)$ for all Weyl groups of ranks less than 6 .

We will now describe examples of the sets $\sigma(W)$. 
5.1. $A_{n}, n \leq 6$. Here $\sigma(W)=W$. Thus, all intersection cohomology complexes with coefficients in an arbitrary field have the same characters as in characteristic zero and the decomposition theorem is true with field coefficients of any characteristic. This is the statement of Theorem 1.3.

5.2. $A_{7}$. Let $W=A_{7}$ with Coxeter generators $s_{i}$ with $i \in\{1, \ldots, 7\}$ corresponding to the simple transpositions $(i, i+1)$. In $W, 38$ of the 40320 elements do not belong to $\sigma(W)$. The elements which do not lie in $\sigma(W)$ break up naturally into five groups, which we now describe.

Consider the following elements of $W$ :

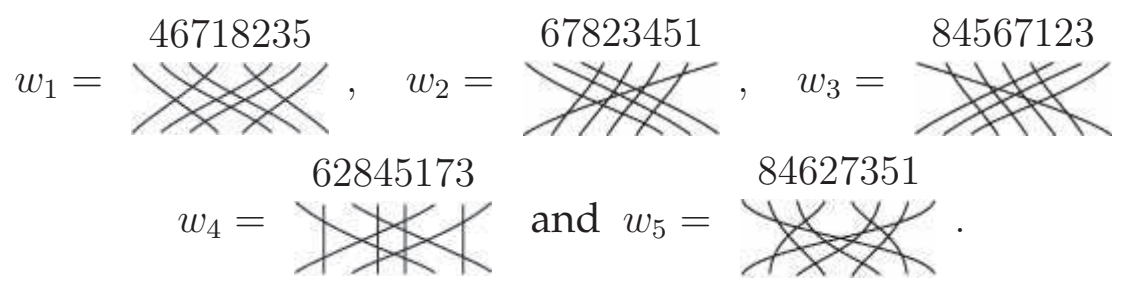

The first group consists of

$$
K_{1}=\left\{u w_{1} v \mid u, v \in\left\langle s_{4}\right\rangle\right\} .
$$

The second group consists of

$$
K_{2}=\left\{w_{2}, s_{5} w_{2}, s_{1} w_{2}, s_{1} s_{5} w_{2}, w_{2} s_{3}, w_{2} s_{7}, w_{2} s_{3} s_{7}\right\} .
$$

(note that $w_{2}$ is maximal in $K_{2}$ ). The third group $K_{3}$ is obtained from $K_{2}$ by inversion (or by applying the automorphism $s_{i} \mapsto s_{8-i}$ ). It contains $w_{3}$ as a maximal element. The fourth group consists of

$$
K_{4}=\left\{u w_{4} v \mid u, v \in\left\langle s_{2}, s_{6}\right\rangle\right\} .
$$

The fifth group consists of

$$
K_{5}=\left\{u w_{5} v \mid u, v \in\left\langle s_{4}\right\rangle\right\} .
$$

It would be interesting to investigate the intersection cohomology complexes corresponding to the minimal elements in $K_{i}$ for $i \neq 1$ directly.

Note that the set $K_{1}$ has already arisen in Kazhdan-Lusztig combinatorics; these are the so-called "hexagon permutations" of Billey and Warrington (see [BW01], the name refers to a characteristic hexagon shape appearing in their heap representation).

The significance of these permutations is explained by the following result. We say that a permutation $w \in S_{n}$ contains the pattern of a permutation $y \in S_{m}$ if there is a collection of indices $1 \leq i_{1}<$ 
$\cdots<i_{m} \leq n$ so that $w\left(i_{1}\right), \ldots, w\left(i_{m}\right)$ are in the same relative order as $y(1), \ldots, y(m)$. Otherwise, we say that $w$ avoids the pattern $y$.

Theorem 5.1. ([BW01, Theorem 1]) Let $\mathbf{w}$ be a reduced word for an element $w \in S_{n}$. Then the corresponding Bott-Samelson resolution $\pi: \Sigma_{\mathbf{w}} \rightarrow$ $X_{w}$ (see the appendix) is a small map if and only if the permutation $w$ avoids the pattern 321 and the four hexagon permutations $w_{1}, s_{4} w_{1}, w_{1} s_{4}$ and $s_{4} w_{1} s_{4}$.

For a more general notion of pattern avoidance which works for general Coxeter groups, see [BP05]. A geometric interpretation of pattern avoidance is given in [BB03].

The Bott-Samelson resolutions of the hexagon permutations are semi-small, but not small (as can be checked directly). In the appendix, Braden treats the intersection cohomology complex over $\mathbb{Z}$ corresponding to $w \in K_{1}$ in detail, and shows that they have 2-torsion in their costalks. In particular, $\operatorname{ch}(\mathcal{E}(w, \mathbb{k})) \neq \underline{H}_{w}$ for $w \in K_{1}$ if $\mathbb{k}$ is a field of characteristic 2 by Corollary 3.13.

5.3. $B_{2}$ and $B_{3}$. As $B_{2}$ is dihedral, it has already been covered in Example 4.1 where we saw that, if $W=\left\langle s, t \mid(s t)^{4}=1\right\rangle$ then $W \backslash$ $\sigma(W)=\{s t s, t s t\}$. One calculates easily that

$$
\underline{B}_{s t s}=\underline{H}_{s t s}+\underline{H}_{s} \quad \text { and } \quad \underline{B}_{t s t}=\underline{H}_{t s t}+\underline{H}_{t} .
$$

One may show that, if $s$ (resp. $t$ ) denotes the simple reflection corresponding to the long (resp. short) simple root then the Schubert variety corresponding to sts is smooth and so $\mathcal{E}(s t s) \cong \mathbf{I C}_{B}($ sts) for any field of coefficients. Furthermore one may show (either by direct calculation or using the results of [JW]) that $\mathcal{E}(t s t) \cong \mathbf{I C}_{B}(t s t)$ if and only if the coefficients $\mathbb{k}$ are not of characteristic 2 . It follows from Proposition 4.3 that, with coefficients of characteristic 2 , one has

$$
\operatorname{ch}(\mathcal{E}(t s t))=\underline{H}_{t s t}+\underline{H}_{t} .
$$

Now consider $W=B_{3}$ with generators

$$
s-t=v \text {. }
$$

In this example, $\underline{B}_{x}$ is an $\mathbb{N}$-linear combination of Kazhdan-Lusztig basis elements for all $x \in W$ except $x=$ stsuts, where one has

$$
\underline{B}_{\text {stsuts }}=\underline{H}_{\text {stsuts }}+\left(v+v^{-1}\right) \underline{H}_{s t s} \text {. }
$$

Of the 48 elements of $W, 21$ do not lie in $\sigma(W)$. They are:

$$
\begin{gathered}
\text { utu, tut, utsu, tuts, utsut, tsuts, sutu,tsutu, } \\
\text { utsutu, }(\text { tsu })^{2}, \text { tutsutu, stut, stsut, stsuts, }(\text { sut })^{2}, \text { sutsutu, } \\
\text { tsutsut, tsutsutu, stuts, stutsutu, stsutsut. }
\end{gathered}
$$


5.4. $B_{4}$ and $B_{5}$. In $B_{4}$, which contains 384 elements, 221 elements do not lie in $\sigma(W)$. In $B_{5}$, which contains 3840 elements, 2627 elements do not lie in $\sigma(W)$.

5.5. $C_{2}, C_{3}, C_{4}$ and $C_{5}$. Note that this is covered by the discussion of type $B$ above.

5.6. $D_{4}$. We label our generators $s, t, u$ and $v$ of $W$ as follows:

$$
s-t^{\prime}{ }_{v}^{u}
$$

Here, 7 elements do not belong to $\sigma(W)$. Let $\tau$ be the automorphism of $W$ mapping $s \mapsto u \mapsto v \mapsto s$. The elements not in $\sigma(W)$ are $w_{1}=$ tvtsutv, $\tau\left(w_{1}\right)$ and $\tau^{2}\left(w_{1}\right)$ as well as $w_{2}=$ suvtvsu, $t w_{2}, w_{2} t$ and $t w_{2} t$. In the appendix, Braden discusses the case of $w_{2}$ in more detail.

5.7. $D_{5}$ and $D_{6}$. In $D_{5}, 171$ of the 1920 elements in $W$ do not lie in $\sigma(W)$. In $D_{6}$, which contains 23040 elements, 3713 elements of $W$ do not lie in $\sigma(W)$.

5.8. $F_{4}$. In $F_{4}, 949$ of the 1152 elements do not lie in $\sigma(W)$.

5.9. $G_{2}$. In this case we have already calculated $\sigma(W)$ in Example 4.1. Here we obtain nothing new. If $W=\left\langle s, t \mid s^{2}=t^{2}=(s t)^{6}=1\right\rangle$ then $\sigma(W)=\{1, s, t, s t, t s$, ststst $\}$. However these Schubert varieties are smooth (this is obvious for $X_{\text {ststst }}=G / B$ and for the others it follows because the Bott-Samelson resolution is an isomorphism), and so $\operatorname{ch}(\mathbf{I C}(w, \mathbb{k}))=\operatorname{ch}(\mathcal{E}(w, \mathbb{k}))=\underline{H}_{w}$ in any characteristic if $w \in$ $\sigma(W)$.

5.10. Further calculations. Let us briefly describe how the above algorithm may be taken further with some of the geometric input contained in the appendix.

For example, if $W$ is of type $A_{7}$ then, with notation as in 5.2, the calculations in the appendix allow one to conclude that $\operatorname{ch}\left(\mathcal{E}\left(w_{1}\right)\right)=$ $\underline{H}_{w_{1}}$ if char $\mathbb{k} \neq 2$. Then the above algorithm may be used to deduce that $\operatorname{ch}(\mathcal{E}(w))=\underline{H}_{w}$ for all hexagon permutations $w$.

Similarly in $D_{4}$ if one knows, with notation as in 5.6, that $\operatorname{ch}\left(\mathcal{E}\left(w_{2}\right)\right)=$ $\underline{H}_{w_{2}}$ then it follows that $\mathcal{E}\left(t w_{2}\right), \mathcal{E}\left(w_{2} t\right)$ and $\mathcal{E}\left(t w_{2} t\right)$ all have characters are given by Kazhdan-Lusztig basis elements. By the calculations of the appendix this occurs if and only if char $\mathbb{k} \neq 2$.

One can sometimes turn these arguments around to deduce that $\operatorname{ch}(\mathcal{E}(w)) \neq \underline{H}_{w}$ for all $w \in K$ for some subset $K \subset W$, once one 
knows that this is the case for one $w \in K$. For example, if $W$ is of type $D_{4}$ and one knows that $\operatorname{ch}\left(\mathcal{E}\left(w_{2}\right)\right) \neq \underline{H}_{w_{2}}$ (as is the case in characteristic 2) one cannot have $\operatorname{ch}(\mathcal{E}(w))=\underline{H}_{w}$ for $w \in\left\{t w_{2}, w_{2} t, t w_{2} t\right\}$. Indeed, let us assume, for example, that $\operatorname{ch}\left(\mathcal{E}\left(t w_{2}\right)\right)=\underline{H}_{t w_{2}}$. Using the $W$-graph one may calculate

$$
\underline{H}_{s} \underline{H}_{t w_{2}}=\underline{H}_{s t s u v}+\underline{H}_{s u t v t s u}+\underline{H}_{s t s v t s u}+\underline{H}_{s t s u t s v}+\underline{H}_{s t s u t v t s u} .
$$

Now, $s t w_{2}=$ stsutvtsu is separated, and hence $\operatorname{ch}\left(\mathcal{E}\left(s t w_{2}\right)\right)=\underline{H}_{s t w_{2}}$. It follows that we have a decomposition

$$
\vartheta_{s}\left(\mathcal{E}\left(t w_{2}\right)\right)=\mathcal{E}\left(s t w_{2}\right) \oplus \mathcal{E}^{\prime}
$$

with $\mathcal{E}\left(w_{2}\right)$ occuring as a direct summand of $\mathcal{E}^{\prime}$. It follows by Lemma 4.2 that

$$
\operatorname{ch}\left(\mathcal{E}\left(w_{2}\right)\right) \leq \underline{H}_{s t s u v}+\underline{H}_{w_{2}}+\underline{H}_{s t s v t s u}+\underline{H}_{s t s u t s v} .
$$

However, by Proposition 4.3,

$$
\operatorname{ch}\left(\mathcal{E}\left(w_{2}\right) \leq \underline{B}_{w_{2}}=\underline{H}_{w_{2}}+\underline{H}_{\text {suv }} .\right.
$$

Hence $\operatorname{ch}\left(\mathcal{E}\left(w_{2}\right)\right)=\underline{H}_{w_{2}}$ as well. Analogous arguments apply in the other cases.

Similarly one may show in type $A_{7}$ that if $\operatorname{ch}\left(\mathcal{E}_{w}\right) \neq \underline{H}_{w}$ for some hexagon permutation $w$, then $\operatorname{ch}\left(\mathcal{E}_{x}\right) \neq \underline{H}_{x}$ for all hexagon permutations. A geometric explanation for this is given in Remark A.17 of the appendix.

\section{APPENDIX A. EXAMPLES OF TORSION IN $I H$ OF SCHUbERT VARIETIES IN TYPES $A_{7}$ AND $D_{4}$}

\section{by Tom Braden ${ }^{6}$}

Let $G$ be a connected reductive complex group, and fix a choice of a Borel subgroup $B$, maximal torus $T$, and opposite Borel subgroup $B^{-}$, so $T=B \cap B^{-}$. Let $\Phi^{+} \subset \Phi \subset X(T)$ be the corresponding sets of roots and positive roots, chosen so that the weights of $\operatorname{Ad} T$ acting on $\mathfrak{b}$ are $-\Phi^{+} \cup\{0\}$. Let $W=N(T) / T$ denote the Weyl group and $S \subset W$ the set of simple reflections. Let $\ell: W \rightarrow \mathbb{N}$ be the length function, and $\leq$ the Bruhat-Chevalley order on $W$.

Consider the flag variety $X=G / B ; G$ acts on $X$ by left multiplication. The set of $T$-fixed points is in bijection with $W$ by $w \mapsto \tilde{w} B / B$, where $\tilde{w}$ is any lift of $w$ to $G$. Using this bijection, we abuse notation and refer to points in $X^{T}$ and elements of $W$ by the same symbols.

\footnotetext{
${ }^{6}$ supported in part by National Science Foundation grant DMS-0201823 and NSA grant H98230-08-1-0097
} 
The flag variety $X$ has two decompositions by Bruhat cells and dual Bruhat cells $X=\coprod_{w \in W} X_{w}=\coprod_{w \in W} S_{w}$, where $X_{w}=B \cdot w$ and $S_{w}=B^{-} \cdot w$. The dual cell $S_{y}$ is a normal slice at $y \in X_{y}$ to the stratification $\left\{X_{w}\right\}_{w \in W}$. This is a consequence of the following lemma, which we will also use in our analysis of Bott-Samelson varieties.

Let $N, N^{-}$be the unipotent parts of $B, B^{-}$. Take $y \in W$, and define $N_{y}=y N^{-} y^{-1}$ and $N_{y}^{+}=N_{y} \cap N$. Then $N_{y}^{+}$is a connected unipotent group of dimension $\ell(w)$.

Lemma A.1. The map

$$
N_{y}^{+} \times S_{y} \rightarrow X, \quad(g, x) \mapsto g \cdot x
$$

is an $N_{y}^{+}$-equivariant isomorphism onto a T-invariant Zariski neighborhood of $y$. This isomorphism trivializes the stratification by Schubert cells $X_{w}$ in the sense that all strata of the induced stratification on $N_{y}^{+} \times S_{y}$ are of the form $N_{y}^{+} \times S$ for some $S \subset S_{y}$. The set $N_{y}^{+} \times\{y\}$ is one such stratum, and it is mapped isomorphically onto $X_{y}$.

Proof. The map $\phi: N_{y} \rightarrow X$ sending $g$ to $g \cdot y$ is an isomorphism onto a $T$-invariant neighborhood $U$ of $y$. It identifies $N_{y}^{+}$with $X_{y}$ and $N_{y}^{-}$ with $S_{y}$, where we put $N_{y}^{-}=N_{y} \cap N^{-}$. Since the multiplication map $N_{y}^{+} \times N_{y}^{-} \rightarrow N_{y}$ is an isomorphism of varieties, we get isomorphisms

$$
N_{y}^{+} \times S_{y} \cong N_{y}^{+} \times N_{y}^{-} \cong N_{y} \cong U
$$

The $N_{y}^{+}$-equivariance is obvious, and the remaining statements about the stratification follow easily from this.

It is easy to describe the one-dimensional $T$-orbits in $X$. The closure of a one-dimensional orbit is a closed irreducible $T$-invariant curve; we will refer to such curves as "T-curves" for short. For any positive root $\mu \in \Phi^{+}$(simple or not), let $s_{\mu} \in W$ denote the corresponding reflection.

Proposition A.2. For any $\mu \in \Phi^{+}$and any $w \in W$, there is a unique $T$-curve $C$ which contains $w$ and $w s_{\mu}$, and all T-curves are of this form. The weight of the action of $T$ on the tangent space $T_{w} C$ is $w(\mu)$.

Since $s_{w(\mu)}=w s_{\mu} w^{-1}$, the above formula for the tangent weight can also be given, up to sign, by saying that if $C$ is the $T$-curve joining $w$ and $s_{\mu^{\prime}} w$ for some $\mu^{\prime} \in \Phi^{+}$, then the $T$-weight of $T_{w} C$ is $\pm \mu^{\prime}$. The sign can then be specified by noting that the weight is in $\Phi^{+}$if and only if $w \leq s_{\mu^{\prime}} w$. 
The Bott-Samelson variety. Let $\boldsymbol{\alpha}=\left(\alpha_{1}, \alpha_{2}, \ldots, \alpha_{l}\right)$ be a sequence of simple roots, not necessarily distinct, and let $\mathbf{w}=\left(s_{1}, s_{2}, \ldots, s_{l}\right)$ be the corresponding sequence of simple reflections: $s_{i}=s_{\alpha_{i}}$. Put $w=s_{1} s_{2} \cdots s_{l}$. Then $\mathbf{w}$ is a reduced word for $w$ if $\ell(w)=l$.

For each simple reflection $s_{i}$, let $P_{i}$ be the corresponding minimal parabolic containing $B$, whose Lie algebra is $\mathfrak{b} \oplus \mathfrak{g}_{\alpha_{i}}$. The BottSamelson variety $\Sigma_{\mathrm{w}}$ associated to $\mathrm{w}$ is defined to be the quotient

$$
\left(P_{1} \times P_{2} \times \cdots \times P_{l}\right) / B^{l},
$$

where $B^{l}=B \times \cdots \times B$ acts on $P_{1} \times P_{2} \times \cdots \times P_{l}$ on the right by

$$
\left(x_{1}, x_{2}, \ldots, x_{l}\right) \cdot\left(b_{1}, b_{2}, \ldots, b_{l}\right)=\left(x_{1} b_{1}, b_{1}^{-1} x_{2} b_{2}, \ldots, b_{l-1}^{-1} x_{l} b_{l}\right) .
$$

Let $\left[x_{1}, \ldots, x_{l}\right]$ denote the point of $\Sigma_{\mathbf{w}}$ corresponding to $\left(x_{1}, \ldots, x_{l}\right) \in$ $P_{1} \times \cdots \times P_{l}$.

Let $B$ act on $\Sigma_{\mathbf{w}}$ by

$$
b \cdot\left[x_{1}, \ldots, x_{l}\right]=\left[b x_{1}, x_{2}, \ldots, x_{l}\right] .
$$

For any $1 \leq k \leq l$, define a $B$-equivariant map $\pi_{k}: \Sigma_{\mathbf{w}} \rightarrow X$ by $\pi_{k}\left(\left[x_{1}, \ldots, x_{l}\right]\right)=x_{1} \cdots x_{k} B$. Let $\pi=\pi_{l}$.

We can filter $\Sigma_{\mathbf{w}}$ by smaller Bott-Samelson varieties as follows. For any subset $I \subset\{1, \ldots l\}$, the $B$-invariant subvariety

$$
\left\{\left[x_{1}, \ldots, x_{l}\right] \in \Sigma \mid x_{i}=1 \text { if } i \notin I\right\}
$$

is clearly isomorphic to $\Sigma_{\mathbf{w}_{I}}$, where the word $\mathbf{w}_{I}$ is given by the simple reflections $s_{i}, i \in I$ taken in order of increasing $i$.

Remark A.3. There is a decomposition of $\Sigma_{\mathbf{w}}$ into $2^{l}$ cells so that all of the subvarieties $\Sigma_{\mathbf{w}_{I}}$ are closures of cells. However, this decomposition is not compatible with the projection $\pi$ : the inverse image $\pi^{-1}\left(S_{w}\right)$ is not generally a union of cells. It will be more useful for us to consider a different paving of $\Sigma_{\mathbf{w}}$ by Białynicki-Birula cells below.

The following easy result will be useful when we analyze the fibers of the Bott-Samelson map.

Lemma A.4. For any $y \in Y$ the inverse image $\pi^{-1}\left(S_{y}\right)$ of the dual cell through $y$ is smooth. In addition, if $I \subset\{1, \ldots, l\}$, then $\Sigma_{\mathbf{w}_{I}}$ intersects $\pi^{-1}\left(S_{y}\right)$ transversely.

Proof. Lemma A.1 implies that the action map $N_{y}^{+} \times \pi^{-1}\left(S_{y}\right) \rightarrow \Sigma_{\mathbf{w}}$ is an isomorphism onto an open subset of $\Sigma_{\mathbf{w}}$. It follows that $\pi^{-1}\left(S_{y}\right)$ is smooth and meets any smooth $N_{y}^{+}$-invariant subvariety transversely. 
Fixed points. We next describe the set $\left(\Sigma_{\mathbf{w}}\right)^{T}$ of $T$-fixed points. Let $D_{l}=(\mathbb{Z} / 2 \mathbb{Z})^{l}=\{0,1\}^{l}$. For each $\varepsilon=(\varepsilon(1), \ldots, \varepsilon(l)) \in D_{l}$, define $p(\varepsilon)=p_{\mathbf{w}}(\varepsilon) \in \Sigma_{\mathbf{w}}$ and $\mathbf{w}^{\varepsilon} \in W$ by

$$
p(\varepsilon)=\left[\tilde{s}_{1}^{\varepsilon(1)}, \ldots, \tilde{s}_{l}^{\varepsilon(l)}\right], \mathbf{w}^{\varepsilon}=s_{1}^{\varepsilon(1)} \cdots s_{l}^{\varepsilon(l)} .
$$

For any $\varepsilon \in D_{l}$ and any $1 \leq k \leq l$, define $\varepsilon[k]=(\varepsilon(1), \ldots, \varepsilon(k), 0 \ldots, 0) \in$ $D_{l}$. As usual, we will refer to elements of $W$ and points of $X^{T}$ by the same symbols.

Proposition A.5. The map $\varepsilon \mapsto p(\varepsilon)$ is a bijection between $D_{l}$ and $\left(\Sigma_{\mathbf{w}}\right)^{T}$. For any $\varepsilon \in D_{l}$, we have $\pi_{k}(p(\varepsilon))=\mathbf{w}^{\varepsilon[k]}$, and, in particular, $\pi(p(\varepsilon))=$ $\mathbf{w}^{\varepsilon}$.

Example A.6. Let $G=G L(3, \mathbb{C})$. There are two simple roots, call them $\rho_{1}$ and $\rho_{2}$.

Let $\mathbf{w}=\left(s_{\rho_{1}}, s_{\rho_{2}}, s_{\rho_{1}}, s_{\rho_{2}}, s_{\rho_{1}}\right)$. Let $w_{0}=s_{\rho_{1}} s_{\rho_{2}} s_{\rho_{1}}$ denote the longest element in $W$. Then there are five T-fixed points in $\pi^{-1}\left(w_{0}\right)$, namely $p(\varepsilon)$, where

$$
\varepsilon \in\{11100,01110,00111,10011,11001\} .
$$

Let us denote these five elements of $D_{5}$ by $\varepsilon_{1}, \ldots, \varepsilon_{5}$. There exists a $T$-curve containing $p\left(\varepsilon_{i}\right)$ and $p\left(\varepsilon_{j}\right)$ if and only if $i=j \pm 1 \bmod 5$. For $i=1, \ldots, 5$, the tangent weight at $p\left(\varepsilon_{i}\right)$ of the T-curve joining $p\left(\varepsilon_{i}\right)$ to $p\left(\varepsilon_{i+1}\right)$ is the ith element of the list $-\rho_{1},-\rho_{2}, \rho_{1}, \rho_{2}+\rho_{1}$, and $\rho_{2}$.

The composition of $\pi_{2}: \Sigma_{\mathbf{w}} \rightarrow X$ with the projection $X \rightarrow G / P_{1} \cong \mathbb{C P}^{2}$ restricts to a birational map $\pi^{-1}\left(w_{0}\right) \rightarrow \mathbb{C P}^{2}$ which identifies $\pi^{-1}\left(w_{0}\right)$ with the blow-up of $\mathbb{C P}^{2}$ at two points. The exceptional fibers are the $T$-curves joining $p\left(\varepsilon_{1}\right)$ to $p\left(\varepsilon_{5}\right)$ and $p\left(\varepsilon_{3}\right)$ to $p\left(\varepsilon_{4}\right)$.

One-dimensional orbits. The one-dimensional $T$-orbits of $\Sigma_{\mathbf{w}}$ are more difficult to classify than the fixed points. Unlike the flag variety $G / B$, Bott-Samelson varieties generally have infinitely many $T$-curves. We will describe a collection of $T$-curves which span the tangent space at each fixed point, but there are in general many other $T$-curves.

Denote the standard basis of $D_{l}$ by $\delta_{i}$, where $\delta_{i}(j)=\delta_{i j}$ is the Kronecker $\delta$-function. For any $\varepsilon \in D_{l}$ and $1 \leq i \leq k$, we have a $T$-curve joining $p(\varepsilon)$ and $p\left(\varepsilon+\delta_{i}\right)$, namely

$$
\left\{\left[\tilde{s}_{1}^{\varepsilon(1)}, \ldots, \tilde{s}_{i-1}^{\varepsilon(i-1)}, x, \tilde{s}_{i+1}^{\varepsilon(i+1)}, \ldots, \tilde{s}_{l}^{\varepsilon(l)}\right] \mid x \in P_{i}\right\} .
$$

This curve projects under $\pi$ to the $T$-curve in $G / B$ which joins w $^{\varepsilon}$ and $\mathbf{w}^{\varepsilon+\delta_{i}}$, and so the tangent weight of this curve at $p(\varepsilon)$ is $\pm \mathbf{w}^{\varepsilon[i-1]}\left(\alpha_{i}\right)$. Note that $T$-curves which project down to fixed points, such as the ones in Example A.6, are not of this type. 
Białynicki-Birula cells. Besides their definition as $B$-orbits, the Bruhat cells $\left\{X_{w}\right\}$ in the flag variety $X$ can also be described as BiałynickiBirula cells for the action of a strictly dominant cocharacter $\zeta: \mathbb{C}^{*} \rightarrow$ $T$. For any $w \in W$, we have

$$
X_{w}=B \cdot w=\left\{x \in X \mid \lim _{t \rightarrow \infty} \zeta(t) \cdot x=w\right\} .
$$

The Bott-Samelson variety $\Sigma_{\mathbf{w}}$ will not in general have finitely many $B$-orbits, but we can still consider its Białynicki-Birula cells (for the same cocharacter $\zeta$ ). Given $\varepsilon \in D_{l}$, we define

$$
\Sigma_{\mathbf{w}, \varepsilon}=\left\{x \in \Sigma_{\mathbf{w}} \mid \lim _{t \rightarrow \infty} \zeta(t) \cdot x=p(\varepsilon)\right\} .
$$

Theorem A.7 ([Gau01, Här]). The dimension of the Białynicki-Birula cell $\Sigma_{\mathbf{w}, \varepsilon}$ is

$$
\begin{array}{r}
\operatorname{dim}_{\mathbb{C}} \Sigma_{\mathbf{w}, \varepsilon}=\#\left\{1 \leq k \leq l \mid \mathbf{w}^{\varepsilon[k]}\left(\alpha_{k}\right) \in-\Phi^{+}\right\} \\
=\#\left\{1 \leq k \leq l \mid \ell\left(\mathbf{w}^{\varepsilon[k]}\right)>\ell\left(\mathbf{w}^{\varepsilon[k]} s_{k}\right)\right\} .
\end{array}
$$

The fibers of the map $\Sigma_{\mathbf{w}, \varepsilon} \rightarrow X_{w}, w=\mathbf{w}^{\varepsilon}$ are affine spaces of dimension

$$
\begin{array}{r}
\operatorname{dim}_{\mathbb{C}} \Sigma_{\mathbf{w}, \varepsilon} \cap \pi^{-1}(w)=\#\left\{1 \leq k \leq l \mid \mathbf{w}^{\varepsilon[k-1]}\left(\alpha_{k}\right) \in-\Phi^{+}\right\} \\
=\#\left\{1 \leq k \leq l \mid \ell\left(\mathbf{w}^{\varepsilon[k-1]}\right)>\ell\left(\mathbf{w}^{\varepsilon[k-1]} s_{k}\right)\right\} .
\end{array}
$$

The cells $\pi^{-1}(w) \cap \Sigma_{\mathbf{w}, \varepsilon}$ for all $\varepsilon$ with $w=\mathbf{w}^{\varepsilon}$ give a paving by affines of $\pi^{-1}(w)$.

As a corollary we obtain the following relation between the cells $\Sigma_{\mathbf{w}, \varepsilon}$ and the sub-Bott-Samelson varieties $\Sigma_{\mathbf{w}_{I}}$. Take $\varepsilon \in D_{l}$, and a subset $I \subset\{1, \ldots, l\}$ and assume that $\varepsilon(i)=1$ implies $i \in I$ for all $i$, so the fixed point $p(\varepsilon)$ lies in the subvariety $\Sigma_{\mathbf{w}_{I}} \subset \Sigma_{\mathbf{w}}$.

Corollary A.8. The cell $\Sigma_{\mathbf{w}, \varepsilon}$ is contained in $\Sigma_{\mathbf{w}_{I}} \subset \Sigma_{\mathbf{w}}$ if and only if

$$
\ell\left(\mathbf{w}^{\varepsilon[i-1]}\right)<\ell\left(\mathbf{w}^{\varepsilon[i-1]} s_{i}\right) \text { for all } i \notin I \text {. }
$$

Proof. Using Theorem A.7, compare the dimension of $\Sigma_{\mathbf{w}, \varepsilon}$ with the dimension of the Białynicki-Birula cell in $\Sigma_{\mathbf{w}_{I}}$ containing $p(\varepsilon)$.

An obstruction to splitting the Bott-Samelson sheaf. Let $\mathbb{k}$ be a field or PID. Fix a word $\mathbf{w}$ as above, and let $A_{\mathrm{w}, \mathbb{k}}=\pi_{*} \mathbb{k}_{\Sigma_{\mathbf{w}}}$ be the pushforward to $X$ of the constant sheaf with coefficients in $\mathbb{k}$. We say that the decomposition theorem holds for $A_{\mathrm{w}, \mathbb{k}}$ if it is isomorphic to a direct sum of shifted intersection cohomology sheaves $\operatorname{IC}\left(\overline{X_{y}} ; \mathbb{k}\right)[s]$.

Let $S_{y}^{\circ}=S_{y} \backslash\{y\}$. Consider the natural homomorphism

$$
\phi_{y, \mathbf{w}, \mathbb{k}}: H^{\bullet}\left(\pi^{-1}\left(S_{y}\right), \pi^{-1}\left(S_{y}^{\circ}\right) ; \mathbb{k}\right) \rightarrow H^{\bullet}\left(\pi^{-1}\left(S_{y}\right) ; \mathbb{k}\right)
$$


of cohomology groups. Because $\pi$ is proper, this is the same as the map obtained by applying hypercohomology to the adjunction morphism $\left.\left(i_{y}\right) ! i_{y}\left(\left.A_{\mathrm{w}, \mathbb{k}}\right|_{S_{y}}\right) \rightarrow A_{\mathrm{w}, \mathrm{k}}\right|_{S_{y}}$, where $i_{y}:\{y\} \rightarrow S_{y}$ is the inclusion. Applying $i_{y}^{*}$ to the adjunction gives a map $i_{y}^{!}\left(\left.A_{\mathrm{w}, \mathbb{k}}\right|_{S_{y}}\right) \rightarrow$ $i_{y}^{*}\left(\left.A_{\mathrm{w}, \mathrm{k}}\right|_{S_{y}}\right)=\left.A_{\mathbf{w}, \mathrm{k}}\right|_{y}$ whose hypercohomology also computes $\phi_{y, \mathbf{w}, \mathbf{k},}$ since there is a cocharacter of $T$ which contracts $S_{y}$ onto $y$ (see [Spr84]).

Proposition A.9. Both the source and target of the homomorphism $\phi_{y, \mathrm{w}, \mathbb{Z}}$ are free $\mathbb{Z}$-modules, and they vanish in odd degrees. Consequently, we have $\phi_{y, \mathbf{w}, \mathbb{k}}=\phi_{y, \mathbf{w}, \mathbb{Z}} \otimes_{\mathbb{Z}} \mathbb{k}$.

Proof. Using the last remark and the properness of $\pi$, we see that the target of $\phi_{y, \mathbf{w}, \mathbb{Z}}$ is isomorphic to the cohomology of the fiber $\pi^{-1}(y)$. Since this fiber has a paving by affines, its cohomology is free and vanishes in odd degrees. The freeness and parity vanishing for the source of $\phi_{y, \mathbf{w}, \mathbb{Z}}$ follows from the isomorphism

$$
H^{k}\left(\pi^{-1}\left(S_{y}\right), \pi^{-1}\left(S_{y}^{\circ}\right) ; \mathbb{k}\right) \cong \operatorname{Hom}_{\mathbb{k}}\left(H_{2 d-k}\left(\pi^{-1}(y) ; \mathbb{k}\right), \mathbb{k}\right),
$$

where $d=\operatorname{dim}_{\mathbb{C}} \pi^{-1}\left(S_{y}\right)=l-\ell(y)$. This in turn follows from the freeness of $H_{2 d-k}\left(\pi^{-1}(y)\right) ; \mathbb{k}$ ), the universal coefficient theorem, and Poincaré duality with supports for the smooth variety $\pi^{-1}\left(S_{y}\right)$ - see [Hat02, Proposition 3.46], for example.

The last part now follows by the universal coefficient theorem.

Proposition A.10. If the decomposition theorem holds for $A_{\mathrm{w}, \mathrm{k}}$, then the cokernel of $\phi_{y, \mathbf{w}, \mathbb{k}}$ is a free $\mathbb{k}$-module.

Proof. For any $w \in W$, the map

$$
\left.\mathbb{H} \bullet\left(i_{y}^{!}\left(\left.\mathbf{I C}\left(\overline{X_{w}} ; \mathbb{k}\right)\right|_{S_{y}}\right)\right) \rightarrow \mathbb{H}^{\bullet}\left(\left.i_{y}^{*} \mathbf{I C}\left(\overline{X_{w}} ; \mathbb{k}\right)\right|_{S_{y}}\right)\right)
$$

is an isomorphism if $y=w$ and is zero if $y \neq w$, by the degree vanishing for intersection cohomology. As noted above the map $\mathbb{H} \cdot\left(i_{y}^{!}\left(\left.A_{\mathbf{w}, \mathbb{k}}\right|_{S_{y}}\right)\right) \rightarrow \mathbb{H}\left(i_{y}^{*}\left(\left.A_{\mathbf{w}, \mathbb{k}}\right|_{S_{y}}\right)\right)$ is equal to $\phi_{y, \mathbf{w}, \mathbb{k},}$, so if the decomposition theorem holds, this map is a direct sum of maps whose cokernels are free.

Remark A.11. Suppose that $\mathbf{w}$ is a reduced word for $w \in W$, and that the resulting map $\pi: \Sigma_{\mathbf{w}} \rightarrow \overline{X_{w}}$ is semi-small. In this case, the map $\phi_{y, \mathbf{w}, \mathbb{k}}$ was previously considered in [dCM02, JMW09], in the guise of an intersection form on the top Borel-Moore homology of $\pi^{-1}(y)$ with $\mathbb{k}$ coefficients. Semi-smallness implies that $\phi_{y, \mathrm{w}, \mathrm{k}}$ vanishes except in one degree, namely $\operatorname{dim}_{\mathbb{C}}\left(\overline{X_{w}} \cap S_{y}\right)=\ell(w)-\ell(y)$, and if the coefficients $\mathbb{k}$ are a field or a complete local principal ideal domain, [dCM02, Theorem 3.3.3] or [JMW09, Theorem 3.5] shows that $\phi_{y, w, k}$ 
is an isomorphism for all $y$ if and only if the decomposition theorem holds with coefficients in $\mathbb{k}$. (Note that the result in [dCM02] is stated for $\mathbb{Q}$ coefficients only, but in fact the arguments work more generally.)

In fact, a straightforward generalization of the argument given in [JMW09, Section 3] shows that, even when $\pi$ is not semi-small, the graded multiplicity with which the parity sheaf $\mathcal{E}(y, \mathbb{k})$ occurs as a direct summand of $A_{\mathrm{w}, \mathbb{k}}$ is given by the graded rank of $\phi_{y, \mathbf{w}, \mathbb{k}}$. Hence, if $\mathbb{k}$ is a field of characteristic $p$, then the multiplicity with which $\mathcal{E}(y, \mathbb{k})$ occurs in $A_{\mathbf{w}, \mathbb{k}}$ will be the same as in characteristic 0 if and only if coker $\phi_{y, \mathbf{w}, \mathbb{k}}$ has no $p$-torsion.

It follows from Proposition A.10 that if $\mathbb{k}$ is a field of characteristic $p$ and the cokernel of $\phi_{y, \mathbf{w}, \mathbb{Z}}$ has $p$-torsion, the decomposition theorem will fail for $A_{\mathrm{w}, \mathbb{k}}$, and so we must have $\operatorname{ch} \mathcal{E}(v, \mathbb{k}) \neq \operatorname{ch} \mathcal{E}(v, \mathbb{Q})=\underline{H}_{v}$ for some $v \in W$. Then Proposition 3.11 and Corollary 3.13 imply that the parity sheaf $\mathcal{E}(v, \mathbb{k})$ is not isomorphic to $\mathbf{I C}_{B}\left(\overline{X_{v}} ; \mathbb{k}\right)$ and either the stalks or costalks of $\mathrm{IC}\left(\overline{X_{v}} ; \mathbb{Z}\right)$ will have $p$-torsion.

In general it is difficult to determine for which $v$ this will happen. It is not hard to see, however, that at least one such $v$ must lie in the interval $[y, w]$, where $w$ is the unique maximum element in the set $\left\{\mathbf{w}^{\varepsilon} \mid \varepsilon \in D_{l}\right\}$. The upper bound comes because $\pi\left(\Sigma_{\mathbf{w}}\right)=\overline{X_{w}}$, so the sheaf $A_{\mathrm{w}, \mathrm{k}}$ is supported on $\overline{X_{w}}$. For the lower bound, notice that the argument of Proposition A.10 still applies if we restrict to the open set $U_{y}:=\bigcup_{z>y} X_{y}$, so we can conclude that the decomposition theorem fails for $\left.A_{\mathrm{w}, \mathrm{k}}\right|_{U_{y}}$.

The following result gives one case where it is possible to be more precise about the relation between $\phi_{y, \mathbf{w}, \mathrm{k}}$ and the stalks and costalks of $\operatorname{IC}\left(\overline{X_{w}}, \mathbb{k}\right)$ for a particular $w$.

Proposition A.12. Let $\mathbf{w}$ be a reduced word for $w \in W$, take $y \leq w$, and let $V=\overline{X_{w}} \cap S_{y}$ and $V^{\circ}=\overline{X_{w}} \cap S_{y}^{\circ}$. Suppose that the map $\pi^{-1}\left(V^{\circ}\right) \rightarrow V^{\circ}$ is small. Let $\mathbf{I C}=\left.\mathbf{I C}(V ; \mathbb{k}) \cong \mathbf{I C}\left(\overline{X_{w}} ; \mathbb{k}\right)\right|_{V}$, shifted so that $\left.\mathbf{I C}\right|_{X_{w} \cap S_{y}}$ is a constant local system in degree 0 . If $d=\operatorname{dim}_{\mathbb{C}} V$, then the stalks and costalks of IC at $y$ are given by

$$
\mathbb{H}^{r}\left(i_{y}^{*} \mathbf{I C}\right)= \begin{cases}\operatorname{coker} \phi^{r} & \text { if } r \in 2 \mathbb{Z} \text { and } r<d \\ \operatorname{ker} \phi^{r+1} & \text { if } r+1 \in 2 \mathbb{Z} \text { and } r<d \\ 0 & \text { otherwise }\end{cases}
$$


and

$$
\mathbb{H}^{r}\left(i_{y}^{!} \mathbf{I C}\right)= \begin{cases}\operatorname{ker} \phi^{r} & \text { if } r \in 2 \mathbb{Z} \text { and } r>d \\ \text { coker } \phi^{r-1} & \text { if } r-1 \in 2 \mathbb{Z} \text { and } r>d \\ 0 & \text { otherwise }\end{cases}
$$

where $\phi=\phi_{y, \mathbf{w}, \mathbb{k}}$ and $\phi^{r}$ is the degree $r$ part of $\phi$.

Proof. Put $i=i_{y}$ and let $j: V^{\circ} \rightarrow V$ be the inclusion. Let $A=\left.A_{\mathbf{w}, \mathbb{k}}\right|_{V}$; it is isomorphic to the pushforward of $\underline{\mathbb{k}}_{\pi^{-1}(V)}$ to $V$. Since the map $\pi^{-1}\left(V^{\circ}\right) \rightarrow V^{\circ}$ is small, we have $j^{*} A \cong \mathbf{I C}\left(V^{\circ} ; \mathbb{k}\right)$.

From the truncation triangle

$$
\mathbf{I C}=\tau_{\leq d-1} j_{*} j^{*} A \rightarrow j_{*} j^{*} A \rightarrow \tau_{\geq d} j_{*} j^{*} A \stackrel{[1]}{\longrightarrow}
$$

it follows that $\mathbb{H}^{r}\left(i^{*} \mathbf{I C}\right)=\mathbb{H}^{r}\left(i^{*} j_{*} j^{*} A\right)$ if $r<d$, and $\mathbb{H}^{r}\left(i^{!} \mathbf{I C}\right)=$ $\mathbb{H}^{r-1}\left(i^{*} j_{*} j^{*} A\right)$ if $r>d$, and they vanish otherwise. (For the second statement, use the fact that $\tau_{\geq d} j_{*} j^{*} A$ is supported at $y$, so $i^{!} \tau_{\geq d} j_{*} j^{*} A \cong$ $i^{*} \tau_{\geq d} j_{*} j^{*}$ A.)

As noted before Proposition A.9, the map $\phi$ is the hypercohomology of the natural map $i^{!} A \rightarrow i^{*} A$, so applying hypercohomology to the triangle

$$
i^{!} A \rightarrow i^{*} A \rightarrow i^{*} j_{*} j^{*} A \stackrel{[1]}{\longrightarrow}
$$

and using Proposition A.9 (parity vanishing) proves the proposition.

Remark A.13. If the fiber $Y:=\pi^{-1}(y)$ is smooth, then by passing to a tubular neighborhood and using excision we can replace the pair $\left(\pi^{-1}\left(S_{y}\right), \pi^{-1}\left(S_{y}^{\circ}\right)\right)$ by $\left(N, N^{\circ}\right)$, where $N$ is the total space of the normal bundle $\mathcal{N}$ to $Y$ in $\pi^{-1}\left(S_{y}\right)$, and $N^{\circ}$ is the complement of the the zero section in $N$. In this case, by the Thom isomorphism theorem we have $H^{\bullet}\left(N, N^{\circ} ; \mathbb{k}\right) \cong H^{\bullet-2 d}(Y ; \mathbb{k}), d=\operatorname{rank} \mathcal{N}$, and the map $\phi_{y, \mathbf{w}, \mathbb{k}}$ can be identified with multiplication by the Euler class $e(\mathcal{N})$.

The hexagon permutation. Now fix $G=G L(8, \mathbb{C})$, so $W$ is the symmetric group on the set $\{1, \ldots, 8\}$. Taking the torus $T$ to be the diagonal matrices, the lattice $X(T)$ of characters is naturally identified with $\mathbb{Z}^{n}$. Let $\beta_{i}$ be the $i$ th standard basis vector of $\mathbb{Z}^{n}$. The roots of $G$ are then the vectors $\rho_{i j}=\beta_{i}-\beta_{j}$, for $1 \leq i, j \leq 8, i \neq j$.

Choose the Borel subgroup $B$ to be the lower triangular matrices. With this choice, the positive roots are $\rho_{i j}$ with $i<j$, and the simple roots are $\rho_{i} \stackrel{\text { def }}{=} \rho_{i, i+1}, i=1, \ldots, 7$. The simple reflection $s_{\rho_{i}}$ corresponding to $\rho_{i}$ is the transposition of $i$ and $i+1$.

We now fix $w=w_{1}$ where $w_{1}$ is the shortest of the "hexagon permutations" introduced in 5.2. The one-line notation of $w$ is 46718235 . 
A reduced word $\mathbf{w}$ for $w$ can be given by the sequence of simple reflections corresponding to the sequence of simple roots

$$
\boldsymbol{\alpha}=\left(\alpha_{1}, \ldots, \alpha_{14}\right)=\left(\rho_{3}, \rho_{2}, \rho_{1}, \rho_{5}, \rho_{4}, \rho_{3}, \rho_{2}, \rho_{6}, \rho_{5}, \rho_{4}, \rho_{3}, \rho_{7}, \rho_{6}, \rho_{5}\right) \text {. }
$$

Reduced words for the other three hexagon permutations are obtained from this by appending $\rho_{4}$ at the beginning or the end, or both.

Let $y=s_{\rho_{2}} s_{\rho_{3}} s_{\rho_{2}} s_{\rho_{5}} s_{\rho_{6}} s_{\rho_{5}}$. It is given in one-line notation as 14327658 .

Theorem A.14. The source and target of the map

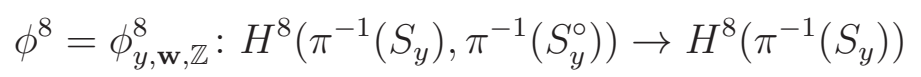

(the degree eight part of $\phi_{y, \mathbf{w}, \mathbb{Z}}$ ) are both isomorphic to $\mathbb{Z}$; its cokernel is isomorphic to $\mathbb{Z} / 2 \mathbb{Z}$.

If 2 is not a unit in $\mathbb{k}$, it follows from this and Proposition A.10 that the decomposition theorem fails with $\mathbb{k}$ coefficients. Furthermore, one can check that the map $\pi: \Sigma_{\mathbf{w}} \rightarrow \overline{X_{w}}$ is a small resolution over the open set $\bigcup_{x>y} X_{x}$, so Proposition A.12 implies that $\operatorname{IC}\left(\overline{X_{w}} ; \mathbb{k}\right)$ has 2-torsion in its costalk at $y$ if $\mathbb{k}=\mathbb{Z}$, and has nonvanishing stalks and costalks in odd degrees if char $\mathbb{k}=2$.

To prove Theorem A.14, we look more closely at the fiber $Y=$ $\pi^{-1}(y)$. We compute the map $\phi^{8}$ using $T$-equivariant cohomology and localization. First we describe the $T$-fixed points in $Y$.

Recall that we denote the standard basis of $D_{l}$ by $\left\{\delta_{i}\right\}$. Define elements of $D_{14}$ by

$$
\begin{array}{ll}
\lambda_{1}=\delta_{1}+\delta_{2}+\delta_{6} & \mu_{1}=\delta_{4}+\delta_{8}+\delta_{9} \\
\lambda_{2}=\delta_{2}+\delta_{6}+\delta_{7} & \mu_{2}=\delta_{8}+\delta_{9}+\delta_{13} \\
\lambda_{3}=\delta_{6}+\delta_{7}+\delta_{11} & \mu_{3}=\delta_{9}+\delta_{13}+\delta_{14} \\
\lambda_{4}=\delta_{7}+\delta_{11}+\delta_{1} & \mu_{4}=\delta_{13}+\delta_{14}+\delta_{4} \\
\lambda_{5}=\delta_{11}+\delta_{1}+\delta_{2} & \mu_{5}=\delta_{14}+\delta_{4}+\delta_{8}
\end{array}
$$

and

$$
\nu=\delta_{5}+\delta_{10} .
$$

Proposition A.15. There are 29 T-fixed points in $Y=\pi^{-1}(y)$. They are given by

(a) $p\left(\lambda_{i}+\mu_{j}\right), 1 \leq i, j \leq 5$, and

(b) $p\left(\lambda_{i}+\mu_{j}+\nu\right), i, j \in\{4,5\}$.

$Y$ has two irreducible components. The first, call it $Y_{1}$, is isomorphic to $Z \times Z$, where $Z$ is isomorphic to $\mathbb{P}^{2}$ blown up at two points. The T-fixed points in $Y_{1}$ are the ones given by (a) above.

The other component $Y_{2}$ is isomorphic to $\mathbb{P}^{1} \times \mathbb{P}^{1} \times \mathbb{P}^{1}$. Its T-fixed points are the four of type (b) and the four of type (a) where $i, j \in\{4,5\}$. 
Remark A.16. We do not need the full statement of the proposition to prove Theorem A.14; we only need the description of the fixed points and the component $Y_{1}$ and the fact that all other components have smaller dimension.

Proof. The enumeration of the points of $Y^{T}$ is straightforward, using Proposition A.5.

Let $I=\{1,2,4,6,7,8,9,11,13,14\}$. Then $w_{I}$ is the (non-reduced) subword of $\mathbf{w}$ corresponding to the sequence of simple roots

$$
\left(\rho_{3}, \rho_{2}, \rho_{5}, \rho_{3}, \rho_{2}, \rho_{6}, \rho_{5}, \rho_{3}, \rho_{6}, \rho_{5}\right) \text {, }
$$

where the roots $\rho_{1}, \rho_{4}$ and $\rho_{7}$ have been omitted from $\mathbf{w}$. As before we identify $\Sigma_{\mathrm{w}_{I}}$ with a subvariety of $\Sigma_{\mathrm{w}}$.

The simple reflections $\rho_{2}, \rho_{3}, \rho_{5}, \rho_{6}$ that appear in $\mathbf{w}_{I}$ generate the Weyl group of the group $G L(3) \times G L(3)$, embedded into $G L(8)$ as block diagonal matrices acting on the middle two factors in the decomposition $\mathbb{C}^{8}=\mathbb{C} \oplus \mathbb{C}^{3} \oplus \mathbb{C}^{3} \oplus \mathbb{C}$. It follows that there is an isomorphism $\Sigma_{\mathbf{w}_{I}} \cong \Sigma_{1} \times \Sigma_{2}$, where the factors are the Bott-Samelson varieties for $\left(\rho_{3}, \rho_{2}, \rho_{3}, \rho_{2}, \rho_{3}\right)$ and $\left(\rho_{5}, \rho_{6}, \rho_{5}, \rho_{6}, \rho_{5}\right)$, respectively. Both $\Sigma_{1}$ and $\Sigma_{2}$ are isomorphic to the Bott-Samelson variety in Example A.6, and it is easy to see that $Y_{1}:=Y \cap \Sigma_{\mathbf{w}_{I}}$ is a product of two copies of the fiber from that example.

To see that $Y_{1}$ is an irreducible component of $Y$, note that a computation with Theorem A.7 shows that the paving by affines of $Y$ given by intersecting with the Białynicki-Birula cells has only one cell of dimension four, namely $Y \cap \Sigma_{\mathbf{w}, \lambda_{1}+\mu_{1}}$, and all other cells are of smaller dimension. So the closure of this cell must be a component of $Y$, and it is the only four-dimensional component, so it is equal to $Y_{1}$.

To understand the other component, note that there is only one cell in $Y \backslash Y_{1}$ of dimension three, namely $\Sigma_{\mathbf{w}, \lambda_{5}+\mu_{5}+\nu} \cap Y$, and all other cells are of smaller dimension. Consider the subword $\mathbf{w}_{J}$, where

$$
J=\{1,2,4,5,7,8,10,11,13,14\} .
$$

It corresponds to the sequence of simple roots

$$
\left(\rho_{3}, \rho_{2}, \rho_{5}, \rho_{4}, \rho_{2}, \rho_{6}, \rho_{4}, \rho_{3}, \rho_{6}, \rho_{5}\right) \text {. }
$$

This is the smallest subword of $\mathbf{w}$ containing all the nonzero entries of $\lambda_{4}, \lambda_{5}, \mu_{4}, \mu_{5}$, and $\nu$. Set $Y_{2}=Y \cap \Sigma_{\mathbf{w}_{J}}$. The $T$-fixed points of $Y_{2}$ are the ones of type (b) and the four of type (a) with $i, j \in\{4,5\}$.

To see that $Y_{2}$ is isomorphic to $\left(\mathbb{P}^{1}\right)^{3}$, we use the following facts, which are easily checked:

- If words $\mathbf{w}_{1}$ and $\mathbf{w}_{2}$ differ by interchanging adjacent transpositions $s_{\rho_{i}}$ and $s_{\rho_{j}}$ with $|i-j|>1$, then the Bott-Samelson 
varieties $\Sigma_{\mathbf{w}_{1}}$ and $\Sigma_{\mathbf{w}_{2}}$ are isomorphic by a $T$-equivariant map which commutes with the projections $\pi_{1}, \pi_{2}$ to $X$.

- If the word $\mathbf{w}_{1}$ is obtained from $\mathbf{w}_{2}$ by doubling $k$ of the simple reflections (i.e. replacing $s_{\rho_{i}}$ with $s_{\rho_{i}} s_{\rho_{i}}$ ), then $\Sigma_{\mathbf{w}_{1}}$ is a fiber bundle over $\Sigma_{\mathbf{w}_{2}}$ with fiber $\left(\mathbb{P}^{1}\right)^{k}$, so that the composition $\Sigma_{\mathbf{w}_{1}} \rightarrow \Sigma_{\mathbf{w}_{2}} \stackrel{\pi_{1}}{\longrightarrow} X$ gives the map $\pi_{2}$.

Using these, if we let $\mathbf{w}_{1}$ and $\mathbf{w}_{2}$ correspond to the sequences

$$
\left(\rho_{3}, \rho_{2}, \rho_{2}, \rho_{5}, \rho_{4}, \rho_{4}, \rho_{3}, \rho_{6}, \rho_{6}, \rho_{5}\right) \text { and }\left(\rho_{3}, \rho_{2}, \rho_{5}, \rho_{4}, \rho_{3}, \rho_{6}, \rho_{5}\right)
$$

of simple roots, then we see that $\Sigma_{\mathbf{w}_{J}} \cong \Sigma_{\mathbf{w}_{1}}$ is a fiber bundle with fiber $\mathbb{P}^{3}$ over $\Sigma_{\mathbf{w}_{2}}$. It is easy to see that the fiber of $\Sigma_{\mathbf{w}_{2}} \rightarrow X$ over $y$ is a single point, so $Y_{2}$, which is the fiber of $\Sigma_{\mathbf{w}_{J}} \rightarrow X$ over $y$, is isomorphic to $\left(\mathbb{P}^{1}\right)^{3}$.

Using Corollary A.8 it is easy to check that all the cells $Y \cap \Sigma_{\mathbf{w}, \varepsilon}$ which are not contained in $Y_{1}$ are contained in $Y_{2}:=Y \cap \Sigma_{\mathbf{w}_{J}}$.

The first part of Theorem A.14 follows immediately. Although the fiber $Y$ is not smooth, there is only one component of dimension four, so the target of $\phi^{8}$ is

$$
H^{8}\left(\pi^{-1}\left(S_{y}\right)\right) \cong H^{8}(Y) \cong H^{8}\left(Y_{1}\right) \cong \mathbb{Z} .
$$

Dually, we have isomorphisms

$$
\begin{aligned}
H^{8}\left(\pi^{-1}\left(S_{y}\right), \pi^{-1}\left(S_{y}^{\circ}\right)\right) & \cong H^{8}\left(\pi^{-1}\left(S_{y}\right), \pi^{-1}\left(S_{y}\right) \backslash Y\right) \\
& \cong H^{8}\left(\pi^{-1}\left(S_{y}\right), \pi^{-1}\left(S_{y}\right) \backslash Y_{1}\right) \cong \mathbb{Z} .
\end{aligned}
$$

Thus we can reduce the computation of $\phi^{8}$ to the smooth case: it is isomorphic to the restriction map $H^{8}\left(N, N \backslash Y_{1}\right) \rightarrow H^{8}(N) \cong H^{8}\left(Y_{1}\right)$, where $N$ is the total space of the normal bundle $\mathcal{N}$ to $Y_{1}$ in $\pi^{-1}\left(S_{y}\right)$. As remarked earlier, this can be identified with the map $H^{0}\left(Y_{1}\right) \rightarrow$ $H^{8}\left(Y_{1}\right)$ given by multiplication by the Euler class $e(\mathcal{N})$, so the image of $\phi^{8}$ is spanned by $e(\mathcal{N})$.

We will compute this class by computing the equivariant Euler class $e_{T}(\mathcal{N}) \in H_{T}^{8}\left(Y_{1}\right)$ and then finding its image in ordinary cohomology. To do this, we split the normal bundle $\mathcal{N}$ into line bundles. We have seen in the proof of Proposition A.15 that $Y_{1}=\Sigma_{\mathbf{w}_{I}} \cap$ $\pi^{-1}\left(S_{y}\right)$. By Lemma A.4 this intersection is transverse, and so $\mathcal{N}$ is isomorphic to the restriction to $Y_{1}$ of the normal bundle to $\Sigma_{\mathbf{w}_{I}}$ in $\Sigma_{\mathbf{w}}$.

Let $I_{1}=I \cup\{3\}, I_{2}=I \cup\{5\}, I_{3}=I \cup\{10\}, I_{4}=I \cup\{12\}$, so $\mathbf{w}_{1}, \ldots \mathbf{w}_{4}:=\mathbf{w}_{I_{1}}, \ldots, \mathbf{w}_{I_{4}}$ are all the subwords of $\mathbf{w}$ of length 11 which contain $\mathbf{w}_{I}$ as a subword. It is easy to see that the subvarieties $\Sigma_{\mathbf{w}_{i}}$ intersect transversely in $\Sigma_{\mathbf{w}}$, so letting $\mathcal{L}_{i}$ be the restriction to 
$Y_{1}$ of the normal bundle to $\Sigma_{\mathbf{w}_{I}}$ in $\Sigma_{\mathbf{w}_{i}}$, we have a splitting $\mathcal{N} \cong$ $\mathcal{L}_{1} \oplus \mathcal{L}_{2} \oplus \mathcal{L}_{3} \oplus \mathcal{L}_{4}$.

To compute the classes $e_{T}\left(\mathcal{L}_{i}\right)$, we compute their restrictions to the fixed point set $Y_{1}^{T}=\left\{p\left(\lambda_{j}+\mu_{k}\right) \mid 1 \leq j, k \leq 5\right\}$. The restriction $\left.e_{T}\left(\mathcal{L}_{i}\right)\right|_{p\left(\lambda_{j}+\mu_{k}\right)} \in H_{T}^{2}\left(p\left(\lambda_{j}+\mu_{k}\right)\right) \cong X(T)$ is just the $T$-weight of the tangent space to the unique $T$-curve containing $p\left(\lambda_{j}+\mu_{k}\right)$, contained in $\Sigma_{\mathbf{w}_{i}}$ and not contained in $\Sigma_{\mathbf{w}}$. This curve is the curve joining $p\left(\lambda_{j}+\right.$ $\left.\mu_{k}\right)$ and $p\left(\eta_{i}+\lambda_{j}+\mu_{k}\right)$, where $\eta_{1}=\delta_{3}, \eta_{2}=\delta_{5}, \eta_{3}=\delta_{10}, \eta_{4}=\delta_{12}$. Using Propositions A.2 and A.5, we can compute that its $T$-weight is the sum of the entries under $\lambda_{j}$ and $\mu_{k}$ in the following tables:

\begin{tabular}{|c|c|c|c|c|c|}
\hline & $\lambda_{1}$ & $\lambda_{2}$ & $\lambda_{3}$ & $\lambda_{4}$ & $\lambda_{5}$ \\
\hline \hline$e_{T}\left(\mathcal{L}_{1}\right)$ & $\rho_{1}+\rho_{2}+\rho_{3}$ & $\rho_{1}+\rho_{2}$ & $\rho_{1}$ & $\rho_{1}$ & $\rho_{1}+\rho_{2}+\rho_{3}$ \\
\hline$e_{T}\left(\mathcal{L}_{2}\right)$ & $\rho_{3}+\rho_{4}$ & $\rho_{4}$ & $\rho_{4}$ & $\rho_{3}+\rho_{4}$ & $\rho_{3}+\rho_{4}$ \\
\hline$e_{T}\left(\mathcal{L}_{3}\right)$ & $\rho_{2}+\rho_{3}+\rho_{4}$ & $\rho_{2}+\rho_{3}+\rho_{4}$ & $\rho_{3}+\rho_{4}$ & $\rho_{3}+\rho_{4}$ & $\rho_{3}+\rho_{4}$ \\
\hline$e_{T}\left(\mathcal{L}_{4}\right)$ & 0 & 0 & 0 & 0 & 0 \\
\hline
\end{tabular}

\begin{tabular}{|c|c|c|c|c|c|}
\hline & $\mu_{1}$ & $\mu_{2}$ & $\mu_{3}$ & $\mu_{4}$ & $\mu_{5}$ \\
\hline \hline$e_{T}\left(\mathcal{L}_{1}\right)$ & 0 & 0 & 0 & 0 & 0 \\
\hline$e_{T}\left(\mathcal{L}_{2}\right)$ & $\rho_{5}$ & 0 & 0 & $\rho_{5}$ & $\rho_{5}$ \\
\hline$e_{T}\left(\mathcal{L}_{3}\right)$ & $\rho_{5}+\rho_{6}$ & $\rho_{5}+\rho_{6}$ & $\rho_{5}$ & $\rho_{5}$ & $\rho_{5}$ \\
\hline$e_{T}\left(\mathcal{L}_{4}\right)$ & $\rho_{5}+\rho_{6}+\rho_{7}$ & $\rho_{6}+\rho_{7}$ & $\rho_{7}$ & $\rho_{7}$ & $\rho_{5}+\rho_{6}+\rho_{7}$ \\
\hline
\end{tabular}

The equivariant class $e_{T}(\mathcal{N})=e_{T}\left(\mathcal{L}_{1}\right) e_{T}\left(\mathcal{L}_{2}\right) e_{T}\left(\mathcal{L}_{3}\right) e_{T}\left(\mathcal{L}_{4}\right)$ induces the same class in $H^{8}\left(Y_{1}\right)$ as

$\left(e_{T}\left(\mathcal{L}_{1}\right)-\left(\rho_{1}+\rho_{2}\right)\right)\left(e_{T}\left(\mathcal{L}_{2}\right)-\left(\rho_{3}+\rho_{4}+\rho_{5}\right)\right)\left(e_{T}\left(\mathcal{L}_{3}\right)-\left(\rho_{3}+\rho_{4}+\rho_{5}\right)\right)\left(e_{T}\left(\mathcal{L}_{4}\right)-\left(\rho_{6}+\rho_{7}\right)\right)$,

where we abuse notation and write a weight $\rho_{i} \in X(T)=H_{T}^{2}(p t)$ instead of its pullback under the map $Y_{1} \rightarrow p t$. After a little computation one sees that this class restricts to zero at every point of $\left(Y_{1}\right)^{T}$ except $p\left(\lambda_{3}+\mu_{1}\right)$ and $p\left(\lambda_{1}+\mu_{3}\right)$, where it has the same restriction as $e_{T}\left(\mathcal{T}_{Y_{1}}\right)$, the equivariant Euler class of the tangent bundle to $Y_{1}$. (To compute the localization of $e_{T}\left(\mathcal{T}_{Y_{1}}\right)$ to the fixed points, use the identification of the weights of $T$-curves in Example A.6. Note that the labeling of the fixed points $\varepsilon_{1}, \ldots, \varepsilon_{5}$ in that example corresponds to the labeling of the fixed points $\lambda_{1}, \ldots, \lambda_{5}$ and $\mu_{1}, \ldots, \mu_{5}$ of $\Sigma_{1}$ and $\Sigma_{2}$.) Then the Atiyah-Bott-Berline-Vergne localization formula [AB84, BV82] implies that $e(\mathcal{N})$ is twice a generator of $H^{8}\left(Y_{1}\right)$, completing the proof of Theorem A.14.

Remark A.17. The other hexagon permutations can be shown to have 2 -torsion by a similar computation; we give only the main points. 
Let $\tilde{\mathbf{w}}=\left(s_{4}\right)^{a} \mathbf{w}\left(s_{4}\right)^{b}$ and $\tilde{y}=\left(s_{4}\right)^{a} y\left(s_{4}\right)^{b}$ for $a, b \in\{0,1\}$. The fiber $\tilde{Y}=\pi^{-1}(\tilde{y})$ is still four-dimensional, but now the union of the components of maximal dimension is isomorphic to $Z_{a} \times Z_{b}$, where $Z_{0}=$ $Z$ and $Z_{1}=Z \cup\left(\mathbb{P}^{1} \times \mathbb{P}^{1}\right)$, the union taken so that $\{0\} \times \mathbb{P}^{1}$ is identified with a $T$-curve in $Z$ with trivial normal bundle. The excess intersection formula [Fu198] then implies that the matrix of $\phi^{8}$ is diagonal under the natural bases given by the components of $\tilde{Y}$ (in other words, the components are orthogonal under the intersection form). The normal bundle to the component $Z \times Z$ is the same as before, so we have $\operatorname{det} \phi^{8} \in 2 \mathbb{Z}$.

Torsion example in D4. Let $G=S O(8 ; \mathbb{C})$. We follow the notation of Section 5.6: the simple reflections in $W$ are $s, t, u, v$ where $s, u, v$ all commute with each other. Let $\mathbf{w}$ be the word $(s, u, v, t, s, u, v)$, put $w=\pi(\mathbf{w})$, and let $y=s u v$.

Proposition A.18. The T-fixed points in $Y:=\pi^{-1}(y)$ are

$$
\left\{p(\varepsilon) \in D_{7} \mid \varepsilon(4)=0 \text { and } \varepsilon(i)+\varepsilon(i+4)=1 \text { for } i=1, \ldots, 3\right\} .
$$

The fiber $Y$ is the transverse intersection of $\Sigma_{(s, u, v, s, u, v)} \subset \Sigma_{\mathbf{w}}$ and $\pi^{-1}\left(S_{y}\right)$. It is T-equivariantly isomorphic to $\mathbb{P}^{1} \times \mathbb{P}^{1} \times \mathbb{P}^{1}$, where the T-weights on the three factors are $\rho_{s}, \rho_{u}$, and $\rho_{v}$, respectively.

By Remark A.13, since $Y$ is smooth, we have $H^{\bullet}\left(\pi^{-1}\left(S_{y}\right), \pi^{-1}\left(S_{y}^{\circ}\right) ; \mathbb{k}\right) \cong$ $H^{\bullet-2}(Y)$, and by Remark A.13 the map $\phi=\phi_{y, \mathbf{w}, \mathbb{Z}}$ can be identified with multiplication by $e(\mathcal{L})$ on $H^{\bullet}(Y)$, where $\mathcal{L}$ is the normal bundle to $Y$ in $\pi^{-1}\left(S_{y}\right)$. As in the previous example we compute this by computing the localization of the equivariant class $e_{T}(\mathcal{L})$ to the fixed points $Y^{T}$. We have

$$
\left.e_{T}(\mathcal{L})\right|_{p(\varepsilon)}=\rho_{t}+\varepsilon(1) \rho_{s}+\varepsilon(2) \rho_{u}+\varepsilon(3) \rho_{v},
$$

so $e(\mathcal{L})=\alpha+\beta+\gamma$, where $\alpha, \beta, \gamma \in H^{2}(Y)$ are the pullbacks of a generating class of $H^{2}\left(\mathbb{P}^{1}\right)$ by the three projection maps. Multiplication by this class from $H^{2}(Y)$ to $H^{4}(Y)$ is given by the matrix

$$
\left[\begin{array}{lll}
1 & 1 & 0 \\
1 & 0 & 1 \\
0 & 1 & 1
\end{array}\right]
$$

with respect to the natural monomial basis in $\alpha, \beta, \gamma$. This matrix has determinant -2 , so coker $\phi^{4}$ has 2-torsion.

Just as we saw for the hexagon permutation, if 2 is not a unit in $\mathbb{k}$ Proposition A.10 implies that the decomposition theorem with $\mathbb{k}$ coefficients fails and Proposition A.12 implies that the costalk of 
$\mathrm{IC}\left(\overline{X_{w}} ; \mathbb{k}\right)$ at $y$ does not vanish in odd degrees. Furthermore, if char $\mathbb{k}=$ 2 , the stalk of $\mathbf{I C}\left(\overline{X_{w}} ; \mathbb{k}\right)$ also has nonvanishing odd-degree part.

\section{REFERENCES}

[AB84] M. F. Atiyah and R. Bott. The moment map and equivariant cohomology. Topology, 23(1):1-28, 1984.

[BB03] S. C. Billey and T. Braden. Lower bounds for Kazhdan-Lusztig polynomials from patterns. Transform. Groups, 8(4):321-332, 2003.

[BBD82] A. Běllinson, J. Bernstein, and P. Deligne. Faisceaux pervers. In Analyse et topologie sur les espaces singuliers, I (Luminy, 1981), volume 100 of Astérisque, pages 5-171. Soc. Math. France, Paris, 1982.

[BL94] J. Bernstein and V. Lunts. Equivariant sheaves and functors, volume 1578 of Lecture Notes in Mathematics. Springer-Verlag, Berlin, 1994.

[BM01] T. Braden and R. MacPherson. From moment graphs to intersection cohomology. Math. Ann., 321(3):533-551, 2001.

[BP05] S. Billey and A. Postnikov. Smoothness of Schubert varieties via patterns in root subsystems. Adv. in Appl. Math., 34(3):447-466, 2005.

[BV82] N. Berline and M. Vergne. Classes caractéristiques équivariantes. Formule de localisation en cohomologie équivariante. C. R. Acad. Sci. Paris Sér. I Math., 295(9):539-541, 1982.

[BW01] S. C. Billey and G. S. Warrington. Kazhdan-Lusztig polynomials for 321-hexagon-avoiding permutations. J. Algebraic Combin., 13(2):111-136, 2001.

[dC] F. du Cloux. Coxeter. a computer program available from math.univlyon1.fr/ ducloux/coxeter/coxeter3/english/coxeter3_e.html.

[dC02] F. du Cloux. Computing Kazhdan-Lusztig polynomials for arbitrary Coxeter groups. Experiment. Math., 11(3):371-381, 2002.

[dCM02] M. A. A. de Cataldo and L. Migliorini. The hard Lefschetz theorem and the topology of semismall maps. Ann. Sci. École Norm. Sup. (4), 35(5):759$772,2002$.

[dCM05] M. A. A. de Cataldo and L. Migliorini. The Hodge theory of algebraic maps. Ann. Sci. École Norm. Sup. (4), 38(5):693-750, 2005.

[Fu198] W. Fulton. Intersection theory, volume 2 of Ergebnisse der Mathematik und ihrer Grenzgebiete. 3. Folge. A Series of Modern Surveys in Mathematics [Results in Mathematics and Related Areas. 3rd Series. A Series of Modern Surveys in Mathematics]. Springer-Verlag, Berlin, second edition, 1998.

[FW] P. Fiebig and G. Williamson. The $p$-smooth locus of Schubert varieties. in preparation.

[Gau01] S. Gaussent. The fibre of the Bott-Samelson resolution. Indag. Math. (N.S.), 12(4):453-468, 2001.

[Här] M. Härterich. The T-equivariant cohomology of Bott-Samelson varieties. preprint math. AG/0412337.

[Hat02] A. Hatcher. Algebraic topology. Cambridge University Press, Cambridge, 2002.

[Hum90] J. E. Humphreys. Reflection groups and Coxeter groups, volume 29 of Cambridge Studies in Advanced Mathematics. Cambridge University Press, Cambridge, 1990. 
[JMW09] D. Juteau, C. Mautner, and G. Williamson. Parity sheaves. preprint arXiv:0906.2994, 2009.

[Jut09] D. Juteau. Decomposition numbers for perverse sheaves. Ann. Inst. Fourier (Grenoble), 59(3):1177-1229, 2009.

[JW] D. Juteau and G. Williamson. Kumar's criterion modulo $p$. in preparation.

[Ker83] S. V. Kerov. $W$-graphs of representations of symmetric groups. Zap. Nauchn. Sem. Leningrad. Otdel. Mat. Inst. Steklov. (LOMI), 123:190-202, 1983. Differential geometry, Lie groups and mechanics, V.

[KL79] D. Kazhdan and G. Lusztig. Representations of Coxeter groups and Hecke Algebras. Invent. Math., 53:165-184, 1979.

[KL80] D. Kazhdan and G. Lusztig. Schubert varieties and Poincaré duality. In Geometry of the Laplace operator (Proc. Sympos. Pure Math., Univ. Hawaii, Honolulu, Hawaii, 1979), Proc. Sympos. Pure Math., XXXVI, pages 185203. Amer. Math. Soc., Providence, R.I., 1980.

[LS81] A. Lascoux and M.-P. Schützenberger. Polynômes de Kazhdan \& Lusztig pour les grassmanniennes. In Young tableaux and Schur functors in algebra and geometry (Torun, 1980), volume 87 of Astérisque, pages 249-266. Soc. Math. France, Paris, 1981.

[Lus80] G. Lusztig. Some problems in the representation theory of finite Chevalley groups. In The Santa Cruz Conference on Finite Groups (Univ. California, Santa Cruz, Calif., 1979), volume 37 of Proc. Sympos. Pure Math., pages 313-317. Amer. Math. Soc., Providence, R.I., 1980.

[Lus03] G. Lusztig. Hecke algebras with unequal parameters, volume 18 of CRM Monograph Series. American Mathematical Society, Providence, RI, 2003.

[OK95] M. Ochiai and F. Kako. Computational construction of $W$-graphs of Hecke algebras $H(q, n)$ for $n$ up to 15. Experiment. Math., 4(1):61-67, 1995.

[Soe97] W. Soergel. Kazhdan-Lusztig polynomials and a combinatoric[s] for tilting modules. Represent. Theory, 1:83-114 (electronic), 1997.

[Soe00] W. Soergel. On the relation between intersection cohomology and representation theory in positive characteristic. J. Pure Appl. Algebra, 152(13):311-335, 2000.

[Spr82] T. A. Springer. Quelques applications de la cohomologie d'intersection. In Bourbaki Seminar, Vol. 1981/1982, volume 92 of Astérisque, pages 249273. Soc. Math. France, Paris, 1982.

[Spr84] T. A. Springer. A purity result for fixed point varieties in flag manifolds. J. Fac. Sci. Univ. Tokyo Sect. IA Math., 31(2):271-282, 1984.

GeORDie WiLliamson, Mathematical Institute, University of OXFORD, 24-29 ST GILES', OXFORD, OX1 3LB, UK

E-mail address: geordie.williamson@maths.ox.ac.uk

URL: http://people.math.ox.ac.uk/williamsong

TOM BRADEN, DePt. Of MATHEMATICS AND StATISTICS, UniVERsity OF MASSACHUSETTS, AMHERST

E-mail address: braden@math.umass .edu 
This figure "perm1a.jpg" is available in "jpg" format from: http://arxiv.org/ps/0709.0207v5 
This figure "w2.jpg" is available in "jpg" format from: http://arxiv.org/ps/0709.0207v5 